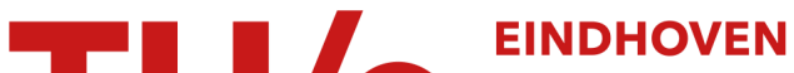 UNIVERSITY OF TECHNOLOGY
}

\section{Physics-based modeling and data representation of pairwise interactions among pedestrians}

\section{Citation for published version (APA):}

Corbetta, A., Meeusen, J. A., Lee, C., Benzi, R., \& Toschi, F. (2018). Physics-based modeling and data representation of pairwise interactions among pedestrians. Physical Review E, 98(6), [062310].

https://doi.org/10.1103/PhysRevE.98.062310

DOI:

10.1103/PhysRevE.98.062310

Document status and date:

Published: 14/12/2018

\section{Document Version:}

Publisher's PDF, also known as Version of Record (includes final page, issue and volume numbers)

\section{Please check the document version of this publication:}

- A submitted manuscript is the version of the article upon submission and before peer-review. There can be important differences between the submitted version and the official published version of record. People interested in the research are advised to contact the author for the final version of the publication, or visit the $\mathrm{DOI}$ to the publisher's website.

- The final author version and the galley proof are versions of the publication after peer review.

- The final published version features the final layout of the paper including the volume, issue and page numbers.

Link to publication

\section{General rights}

Copyright and moral rights for the publications made accessible in the public portal are retained by the authors and/or other copyright owners and it is a condition of accessing publications that users recognise and abide by the legal requirements associated with these rights.

- Users may download and print one copy of any publication from the public portal for the purpose of private study or research.

- You may not further distribute the material or use it for any profit-making activity or commercial gain

- You may freely distribute the URL identifying the publication in the public portal.

If the publication is distributed under the terms of Article 25fa of the Dutch Copyright Act, indicated by the "Taverne" license above, please follow below link for the End User Agreement:

www.tue.nl/taverne

Take down policy

If you believe that this document breaches copyright please contact us at:

openaccess@tue.nl

providing details and we will investigate your claim. 


\title{
Physics-based modeling and data representation of pairwise interactions among pedestrians
}

\author{
Alessandro Corbetta, ${ }^{1}$ Jasper A. Meeusen, ${ }^{1}$ Chung-min Lee, ${ }^{2}$ Roberto Benzi, ${ }^{3}$ and Federico Toschi ${ }^{4}$ \\ ${ }^{1}$ Department of Applied Physics, Eindhoven University of Technology, 5600 MB Eindhoven, The Netherlands \\ ${ }^{2}$ Department of Mathematics and Statistics, California State University, Long Beach, Long Beach, California 90840, USA \\ ${ }^{3}$ Department of Physics and INFN, University of Tor Vergata, I-00133 Rome, Italy \\ ${ }^{4}$ Department of Applied Physics and Department of Mathematics and Computer Science, Eindhoven University of Technology, \\ 5600 MB Eindhoven, The Netherlands \\ and CNR-IAC, I-00185 Rome, Italy
}

(Received 7 August 2018; published 14 December 2018)

\begin{abstract}
In this work we study pedestrian-pedestrian interactions from observational experimental data in diluted pedestrian crowds. While in motion, pedestrians continuously adapt their walking paths trying to preserve mutual comfort distances and to avoid collisions. Leveraging on a high-quality, high-statistics data set, composed of several few millions real-life trajectories acquired from state-of-the-art observational experiments (about 6 months of high-resolution pedestrian tracks acquired in a train station), we develop a quantitative model capable of addressing interactions in the case of binary collision avoidance. We model interactions in terms of both long-range (sight based) and short-range (hard-contact avoidance) forces, which we superimpose on our Langevin model for noninteracting pedestrian motion [Corbetta et al., Phys. Rev. E 95, 032316 (2017)] (here further tested and extended). The model that we propose here features a Langevin dynamics with fast random velocity fluctuations that are superimposed on the slow dynamics of a hidden model variable: the intended walking path. In the case of interactions, social forces may act both on the intended path and on the actual walked path. The model is capable of reproducing quantitatively relevant statistics of the collision avoidance motion, such as the statistics of the side displacement and of the passing speed. Rare occurrences of actual bumping events are also recovered. Furthermore, comparing with large data sets of real-life tracks involves an additional computational challenge so far neglected: identifying automatically, within a database containing very heterogeneous conditions, only the relevant events corresponding to binary avoidance interactions. In order to tackle this challenge, we propose a general approach based on a graph representation of pedestrian trajectories, which allows us to effectively operate complexity reduction for efficient data classification and selection.
\end{abstract}

DOI: 10.1103/PhysRevE.98.062310

\section{INTRODUCTION}

When we walk in a known environment or we explore a new venue, a path is planned in our minds (our "intended path"). As other pedestrians approach us, or as we learn features of the environment (e.g., better directions toward a target destination), this path is continuously adjusted. Either as an impulsive act or as a timely planned adjustment, we sidestep to maintain comfort distances among ourselves and other close by pedestrians. This comes with a modification of our intended walking paths that bend in order to prevent contacts or collisions with others.

The aim of this paper is to quantitatively understand and model the dynamics behind these path changes, in the simplest condition of two pedestrians walking in opposite directions, trying to avoid each other ("pairwise avoidance"; see Fig. 1). This is the first necessary step to understand the interaction physics between pedestrians, before attempting to tackle more complex situations.

The dynamics of path changes is a challenging subject within the broader and compelling issue of understanding the flow of pedestrian crowds [1]. Not only is this scientific topic fascinating because of its connections with the physics of emerging complexity [2], pattern formation [3,4], and active matter [5,6], but it is also extremely relevant for its applications for the design, safety, and performance of civil facilities $[7,8]$.

Because of the macroscopic analogies between crowd and fluid flows [9], modeling pedestrian dynamics in terms of interacting matter particles has become an emerging approach [6]. This analogy underlies proper translations between passive fluid particles, which move under the action of classic interaction forces, and active pedestrians in crowds that interact via social forces [10]. Social forces abstract pedestrianpedestrian interactions in a Newtonian-like way. As such, we expect that mutual repulsive interaction (social) forces may act to ensure comfort distances and collision avoidance, possibly modifying pedestrians' intended paths.

Despite the growing scientific and technological interest for the motion of pedestrian crowds, our quantitative understanding remains relatively limited, especially in comparison to other kinds of active matter systems [11]. A major limitation comes from the fact that high-quality experimental data, with high resolution in space and time, still remain scarce. An important point to be understood is that pedestrian motion has a strong variability, which can be decoupled from average trends only by considering measurements with extremely 


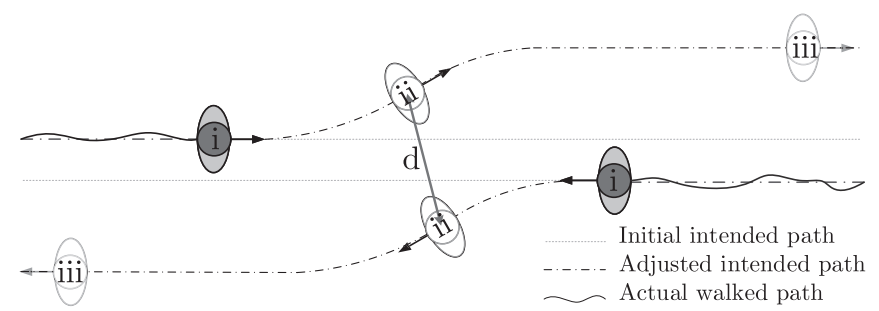

FIG. 1. Mutual avoidance of two pedestrians walking in opposite directions (pairwise avoidance). Pedestrians walk trying to follow straight intended paths (snapshot i), around which they perform random fluctuations (cf. Sec. IV). Individual motions, however, remain influenced by the dynamics of peers. As a peer approaches, the intended path is adjusted (snapshot ii) to ensure maintenance of mutual comfort distance (snapshot iii). We investigate and model quantitatively the avoidance dynamics (cf. Sec. V) with reference to three distances $d$, transversal to the direction of motion, and characteristic of the interaction: (i) before adjusting the intended path (at the entrance of our observation window), (ii) when the two pedestrians are side-by-side, and (iii) when pedestrians leave our observation window.

high statistics. For instance, in the case of a narrow corridor [12], one needs tens of thousands of measured trajectories to estimate the amplitude of the observables' fluctuations (e.g., fluctuations in walking position, velocity, etc.) and to characterize the occurrence of related rare events. In this paper we employ data from tens of thousands of avoidance events to investigate quantitatively and model the changes in intended paths from pairwise avoidance. The measurement of these events was achieved through a months-long real-life experimental campaign that we performed in the main walkway of the train station of Eindhoven, the Netherlands, with state-of-the-art automated pedestrian tracking (see Fig. 2).

The current scarceness of high-quality measurement data is probably related to technical challenges connected to the acquisition of pedestrian trajectory data. Collecting data in reallife conditions demands robust individual tracking techniques, i.e., techniques that remain accurate regardless of factors such as illumination, clothing, presence of objects, crowd density, and so on.

The analysis of pedestrian dynamics in a real-life setting involves, moreover, an additional challenge so far neglected: the automated crowd scenario classification. To illustrate this, let us consider the trajectories collected over weeks in a measurement zone within, e.g., a station or a mall. These trajectories will certainly encompass different and alternating crowd scenarios. For instance, these can include pedestrians walking undisturbed (i.e., with no peers walking in their neighborhood), pedestrians in small or large social groups, diluted or dense crowd streams in counterflows, diluted or dense crowd streams in coflows, and so on (indeed, all these scenarios occur in the measurements considered in this paper; see Fig. 2). If we focus on a given scenario that is defined by a set of parameters (for instance, pedestrians walking in a unidirectional flow at an assigned density level), and we compare the measurements from the occurrences of such a scenario, we expect to observe analogous features up to random fluctuations. Besides, as the number of observed
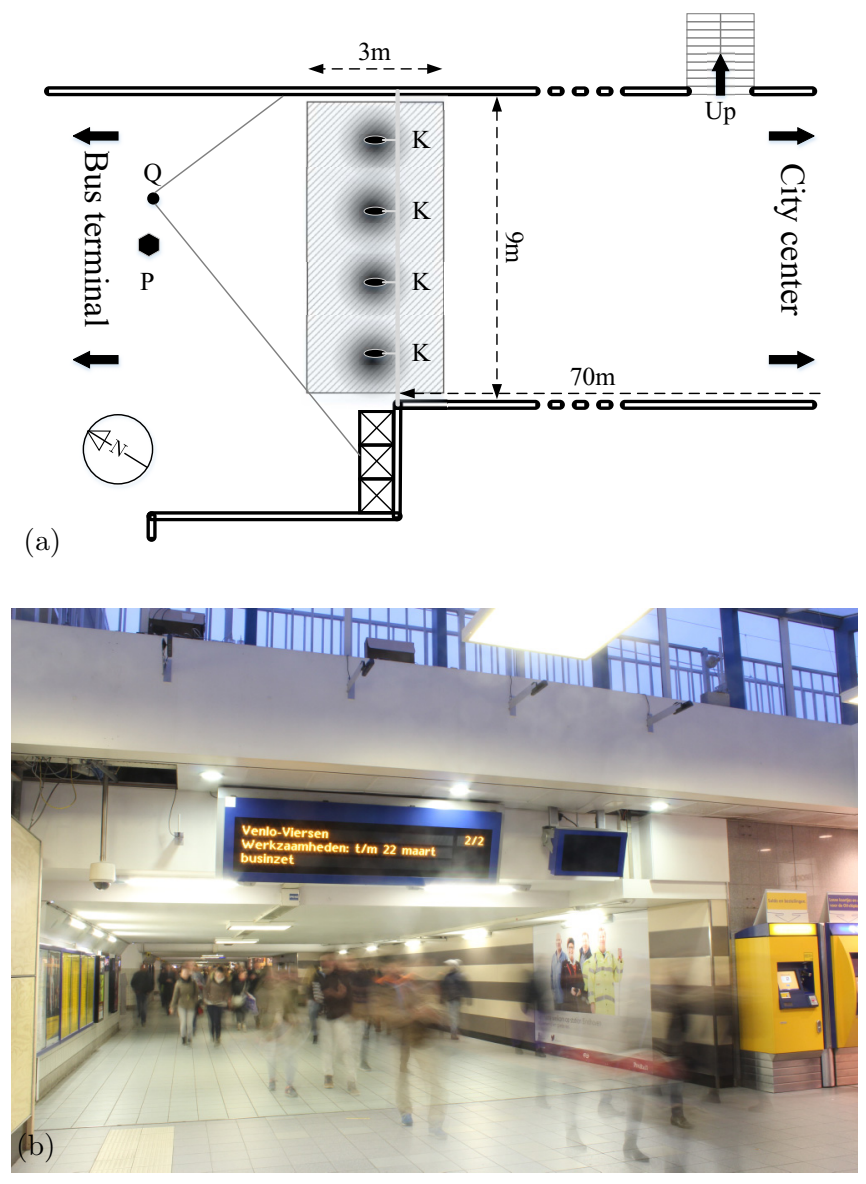

FIG. 2. Experimental setup used in our 6-month-long pedestrian tracking campaign at the train station in Eindhoven, NL; cf. Sec. II. (a) Planar layout of the north entrance hall of Eindhoven train station (as it was between 2013 and early 2015). The measurement area is shaded. The entrance hall, facing the bus terminal of the city, leads to a 70-m-long tunnel connecting to the south side of the city. The rail platforms are reachable from the tunnel via several side staircases. Our pedestrian dynamics recordings employed four overhead Kinect $^{\mathrm{TM}}$ [22] sensors (K) with partially overlapping view. The sensors were attached to the side of the overhang and were supported by metallic arms [cf. (b)]. The snapshot in (b) was taken from point $\mathrm{Q}$, i.e., in the vicinity of a structural pillar (P) (about $5 \mathrm{~m}$ upstream of the recording area).

occurrences increases, we can quantify with higher and higher accuracy the statistics of fluctuations and of the rare events characteristic of the scenario. For instance, people walking undisturbed are expected to have similar speed within fluctuations. If instead we consider social groups, we expect to measure velocities consistently lower than in the undisturbed case [13]. Similarly, we expect counterflow occurrences to exhibit mutual similarities, yet to feature different characteristic fluxes than coflow conditions $[14,15]$. The scenario considered in this paper involves pairs of pedestrians mutually avoiding each other. To analyze the scenario including statistic fluctuations, we aggregate and analyze as an ensemble all the trajectories occurring under such conditions.

More in general, we are interested in subcollections of our observational experimental data pertaining to a given scenario 
of interest, from now on referred to as a selected scenario $\left(S_{\text {sel }}\right)$. Each $S_{\text {sel }}$ is itself defined by a set of control parameters (e.g., number of pedestrians involved, flow conditions considered, etc.). All occurrences of a $S_{\text {sel }}$ in the experimental data set constitute the experimental data, which we investigate as in a traditional laboratory experiment. Differently from a laboratory experiment, the pedestrians involved are not instructed to perform a predefined dynamics (cf. e.g., [16]); rather they can freely walk, without potential biases from the experimental setting. Identifying the subset of trajectories belonging to a target scenario is thus a necessary first step in our investigation. Since we deal with hundreds of thousands of trajectories, this identification cannot be performed manually. In fact, this would demand an exhaustive visual analysis of thousands of hours of sensors' footage (something that has been routinely performed by humans in other smaller scale investigations, e.g., to select groups in Ref. [17], to classify walking patterns in Ref. [18], or to isolate people waiting in Ref. [19]). More in general, this identification task underlies a classification problem in which we associate each trajectory with its scenario.

Automatizing the trajectory classification task is the second aim of this paper, and it is instrumental to analyze the dynamics of path changes. While automatic classification is widely studied in connection with, e.g., images, text, or speech content [20], to the best of our knowledge this topic remains not yet addressed in the context of scenarios made of (pedestrian) trajectories. Once more, this likely relates with the fact that extensive data collection campaigns for pedestrian dynamics remain a rarity.

In this paper we target a twofold state-of-the-art advancement. First, we propose a representation strategy for pedestrian dynamics measurements, based on graphs, to formally identify scenarios and automatically classify and select real-life trajectory data on such a basis. Second, we address quantitatively the dynamics of path changes and related pedestrian-pedestrian social forces in the case of avoidance events involving two individuals (i.e., no third individual plays a role in the dynamics). For this we propose a Langevin-like model, built by extending our previous quantitative model for the diluted (i.e., undisturbed and noninteracting) pedestrian dynamics [12] (cf., e.g., [5,21] for a general modeling reference on Langevin equations). The model is constructed in two steps. First, we generalize the diluted dynamics model to address a richer phenomenology, which is given by a mixture of pedestrians walking and (in tiny percentages) running. Second, we introduce and validate pairwise social forces that act simultaneously on the actual trajectory and on the intended path which we consider a model variable too. This force model enables us to reproduce quantitatively our measurements of the pairwise avoidance dynamics including fluctuations and rare events (actual impacts).

The content of this paper is as follows. In Sec. II we describe our measurement campaign and the acquired data, in the order of millions of trajectories. This sets a basis for both the methodological and the modeling contributions of this work, that are in Sec. III and Secs. IV and V, respectively. In Sec. III we tackle the trajectory selection and classification issue. In Sec. IV we address the motion of undisturbed pedestrians. This is a necessary building block for Sec. V, in which we analyze and model the dynamics of pairwise avoidance and of the intended path. Section VI closes the paper with a summary and final discussion.

\section{MEASUREMENT CAMPAIGN}

The pedestrian dynamics data employed in this work have been collected in the period from October 2014 to March 2015 through a 24/7 real-life campaign at Eindhoven train station. Our data acquisition took place in the initial section of the main walkway of the station as presented in Fig. 2. The walkway is one of the major pedestrian pathways between the north side and the south side of the city with crowd traffic during the entire day. Different dynamics ordinarily occur, such as coflows and counterflows with density ranging from extremely low (one pedestrian in the entire walkway at night time) to high during the morning peak commute times [14]. We aimed at an exhaustive individual tracking with high space and time resolution and overall we collected about 100000 trajectories per day and approximately five millions in total.

Four overhead Microsoft Kinect ${ }^{\mathrm{TM}}$ sensors [22] with partially overlapping view recorded imaginglike data, specifically depth maps, at the rate of 15 frames/s. Depth maps encode in grayscale levels the distance between each filmed pixel and the camera plane; thus regions closer to the overhead sensors, such as heads, result in darker shades. We blend the four depth map signals into a single stream covering the entire measurement region of which we report few frames (already postprocessed to include, e.g., individual trajectories) in Fig. 6. As in our previous investigations (e.g., [23,24]) and following Refs. [25,26], we use cluster-based analyses of depth maps to perform accurate localization of pedestrians' bodies and heads on a frame-by-frame basis. Finally, we employ particle-tracking algorithms to extract individual trajectories from the output localization step. We leave further technical details on the detection and tracking procedures to Appendix A.

\section{REPRESENTATION AND CLASSIFICATION OF CROWD FLOW DATA}

In this section we define two subsets of the trajectories collected in our measurement campaign (cf. Sec. II), which we will use to investigate, respectively, the dynamics of undisturbed flows, considered in Sec. IV, and the dynamics of the pairwise avoidance, considered in Sec. V. These subsets will be the output of a more general representation and classification construct, based on graphs, introduced here.

The underlying issue, as stated in Sec. I, is that large-scale measurements of pedestrian dynamics in real-life conditions typically include different scenarios frequently and randomly changing (see also [27]). For instance, around commuting time, in the walkway in Fig. 2, the flow changes abruptly from diluted to dense. Every few seconds, the typical bidirectional pedestrian flow rapidly turns into a unidirectional dense stream composed of the passengers who had just arrived by train. The scenarios of our interest (undisturbed flow and pairwise avoidance) happen as well, yet alternate at random with others. Instead, we would like to investigate the dynamics of these selected scenarios (cf. Sec. I) separately and 
TABLE I. Bijective correspondences between real-life measurements and their representation in the graph $G$ : summary of concepts and notation (cf. Sec. III).

\begin{tabular}{ll}
\hline \hline Real-life measurement & Graph representation \\
\hline trajectory set $\Gamma$ & graph $G$ \\
trajectory (i.e., pedestrian) $p \in \Gamma$ & node $p \in G$ \\
$p$ and $q$ interacting pedestrians & edge $e=(p, q)$ in $\tilde{G}$ \\
scenario & set of conditions identifying a subgraph $\tilde{G}_{s} \subset \tilde{G}$ \\
realizations of the scenario & connected components in $\tilde{G}_{s}$ \\
pedestrians walking undisturbed (all realizations) & subgraph $\tilde{G}_{1} \subset \tilde{G}$ of the singleton nodes \\
undisturbed pedestrian $p$ (one realization) & $p \in \tilde{G}_{1}$ \\
pedestrians in pairwise avoidance (all realizations) & subgraph $\tilde{G}_{2, a} \subset \tilde{G}$ of dyads with opposite walking direction \\
single pairwise avoidance event of $p$ and $q$ (one realization) & dyad $\{p, q\}$ in $\tilde{G}_{2, a}$ connected by an edge \\
\hline \hline
\end{tabular}

use the data from the realizations of individual scenarios to increment our statistics. For instance, thousands of times per day, pedestrians walking undisturbed cross our measurement area. We expect all of them to exhibit a similar behavior whose statistics we can accurately determine thanks to the large number of trajectories. Analogously, we expect pairs of pedestrians in avoidance (cf. Fig. 1) to show similar features in all realizations of the same dynamics.

In the following subsections we provide a strategy to operate with a $S_{\text {sel }}$. This involves the capability of (i) defining formally and quantitatively a $S_{\text {sel }}$ and (ii) efficiently classifying and aggregating trajectories based on whether or not they are realizations of such a $S_{\text {sel }}$.

In conceptual terms, given the set of measured trajectories, say, the set $\Gamma=\{p\}$, we construct a representation of $\Gamma$ in terms of a graph $G$ with a bijective correspondence between trajectories and graph nodes. This representation, reduced in complexity with respect to the original data set, suitably allows us to define a $S_{\text {sel }}$ as a set of conditions that identify subgraphs of $G$ (Sec. III A). The desired output, i.e., sets of trajectories that are a realization of a $S_{\text {sel }}$, is associated with the connected components of these subgraphs. Trajectories occurring in the same instance of a selected scenario are in the same connected component (and, conversely, the complement of the subgraph identifies trajectories that are not a realization of the given selected scenario).

In Sec. III B we define two subgraphs $\tilde{G}_{1} \subset G$ and $\tilde{G}_{2, a} \subset$ $G$, whose connected components identify, respectively, realizations of diluted flows and of pairwise avoidance, that we will use as experimental comparisons for the models considered, respectively, in Secs. IV and V. In Table I we report a summary of the symbols and concepts used throughout this section.

\section{A. Graph-based representation}

The graph-based representation technique, described in the preceding paragraphs and below, significantly improves what was previously proposed by us in Ref. [27], allowing richer and more parametric scenario classification (the improvement occurs through node annotations and edge weighting). Because of the bijective correspondences between pedestrians and trajectories and between trajectories and graph nodes, in the following we will refer to pedestrians, trajectories, and nodes interchangeably, which we will identify with the generic symbols $p$ and/or $q$.
We build $G$ as follows. We scan in chronological order the set of experimental trajectories (Sec. II) and add a node $p$ to $G$ when a new trajectory is found. We further annotate each node with scalar observables of the trajectory. These are average walking velocity, trajectory length, ultimate direction, and starting and ending positions.

As we scan the trajectory data we also introduce edges between nodes. In particular, if pedestrians $p$ and $q$ appear simultaneously in one or more recorded frames, we add an edge $e=(p, q)$ between the associated nodes.

We vector-weight the edge $e$ with scalars quantifying the pairwise dynamics of $p$ and $q$. The weight $\vec{w}(e)$ reads

$$
\vec{w}(e)=(\min (d), \max (d), \tau),
$$

where $d$ is the distance between $p$ and $q$ (cf. Fig. 1), of which we retain the minimum ( $\min )$ and the maximum (max) observed values, and $\tau$ is the joint recording time, i.e., the duration $p$ and $q$ are both present in our recording window. We report the graph construction algorithm in Fig. 3. We stress that the procedure to construct the graph is efficient in the sense that it is linear in time with the number of frames measured: just one pass of $\Gamma$ is necessary to construct $G$.

\section{B. Flow classification}

The representation via $G$ enables us to formally define a $S_{\text {sel }}$ and efficiently classify observational data in scenarios, both by exploiting the graph topology. In this subsection we introduce the general approach starting from the specific cases of interest for Secs. IV and V.

In Sec. IV we aim at analyzing the dynamics of undisturbed pedestrians. Singleton nodes in $G$ (i.e., edge bereft) provide a first, yet incomplete, collection of these pedestrians. In fact, no other pedestrians potentially perturbing their dynamics were observed while they were crossing our facility (the singleton condition would otherwise be violated).

Singleton nodes of $G$, however, identify just a subset of pedestrians walking undisturbed. In fact, it is reasonable to assume that all individuals remaining sufficiently far from their first neighbor walked undisturbed as well. To correctly classify these cases, heuristically speaking, we remove edges from the nodes that are sufficiently far apart from their neighbors and reduce them to singletons.

More formally, we consider a reference pedestrian $p$ as potentially influenced by a pedestrian $q$, if $q$ enters $p$ 's neighborhood $\mathcal{I}_{p}$ (to be geometrically defined below) for at 


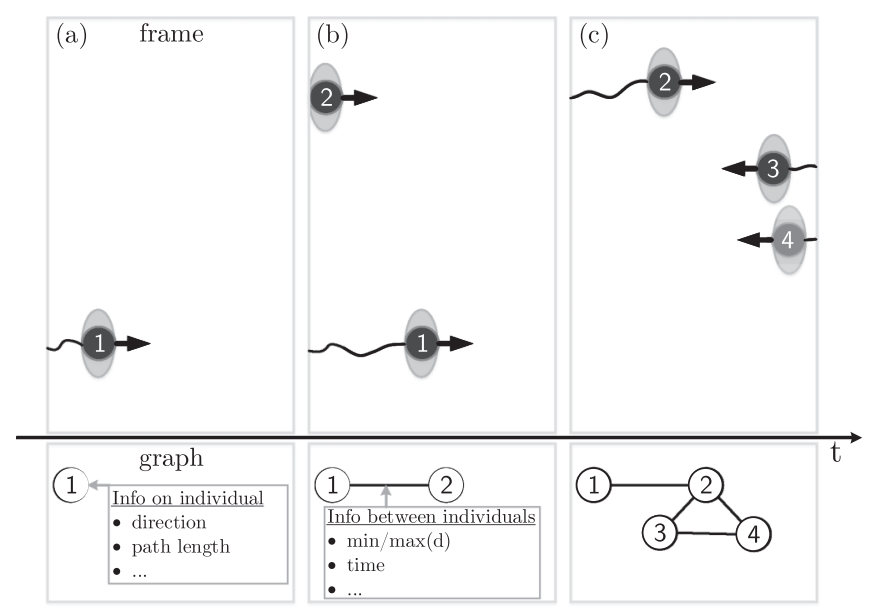

FIG. 3. We represent the recorded crowd dynamics with a graph $G$. This reduced description enables automatic classification of different flow scenarios (cf. Sec. III). Employing the three sample frames (a)-(c), we schematize the graph construction algorithm. As a pedestrian, e.g., pedestrian 1 in (a), appears in our recording window, we add a corresponding node in the graph $G$. When two pedestrians are simultaneously in the recording window, e.g., 1 and 2 in (b), we connect the associated nodes with an edge. As further pedestrians are recorded the graph is expanded. In (c), we imagine that pedestrians 3 and 4 entered the observation window after pedestrian 1 left. Therefore, their nodes are connected to one another and further just with the node representing pedestrian 2 . We include additional information in the graph, crucial for the classification task. Each node is annotated with scalar observables of the associated pedestrian trajectory (e.g., average velocity and direction) and each edge is weighted with scalar observables of the pairwise dynamics of the nodes (e.g., minimum and maximum distances and joint observation time). In the corridor setup considered, annotating the direction on each node means identifying one between two possibilities: either toward the bus terminal or toward the city center (cf. Fig. 2).

least one frame. We define the region $\mathcal{I}_{p}$ (see Fig. 4) considering two criteria: (i) Pedestrians walking at short distances (say, smaller than a given threshold $d_{m}$ ) most likely have an influence on the respective dynamics, therefore

$$
\min (d)<d_{m} \Rightarrow q \in \mathcal{I}_{p}
$$

(ii) pedestrian interactions are anisotropic privileging the motion and sight directions over the transversal directions $[1,28]$. Therefore, letting $d_{y, m}$ be a given threshold for the transversal distance, we set

$$
\min \left(d_{y}\right)<d_{y, m} \Rightarrow q \in \mathcal{I}_{p}
$$

(cf. parameters in Table II). We stress that determining if $q \in$ $\mathcal{I}_{p}$ or $p \in \mathcal{I}_{q}$ consists just of a single check on the vectorweight $\vec{w}(e)$ of the edge $e=(p, q)$.

In the case in which two pedestrians $p$ and $q$, connected by an edge $e$, exert no influence on the motion of each other [according to the metric criteria in Eqs. (2) and (3)], we remove the edge $e$. This operation returns a sparsified subgraph $\tilde{G} \subset G$, likely with an increased number of singletons. Let us call $\tilde{G}_{1}$ the subgraph of singletons of $\tilde{G}$. Here $\tilde{G}_{1}$ identifies all the realizations of undisturbed flows, i.e., the experimental data for our analysis in Sec. IV (further technical constraints on the data set are described in Sec. III C).

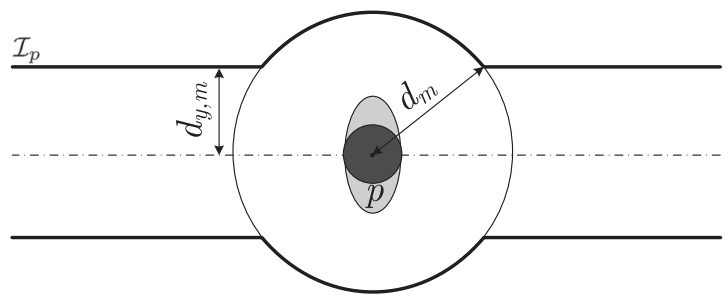

FIG. 4. Sketch of the region $\mathcal{I}_{p}$ around pedestrian $p$. We consider the dynamics of $p$ as potentially being influenced by another pedestrian, say $q$, if, at any time, $q$ entered in $\mathcal{I}_{p}$. Conversely, if, for a time interval $\tau$ no longer than $\tau_{m}, q$ entered in $\mathcal{I}_{p}$ or likewise $p$ entered in $\mathcal{I}_{q}$, we consider the pair $(p, q)$ as noninteracting (even if the two pedestrians appear in the same frames; cf. Fig. 6). By removing the edges in $G$ (cf. $G$ construction in Fig. 3) associated with such noninteracting pedestrians we obtain $\tilde{G}$, in which only potentially interacting pedestrians are connected by edges [cf. Sec. III B, Table I, and Fig. 5(b)]. The region $\mathcal{I}_{p}$ is parametrized by the lengths $d_{m}$ and $d_{y, m}$, the minimum length for interaction and the minimum transversal length for interaction, respectively. (cf. Table II).

As we expect all singleton nodes to be associated with a similar dynamics (undisturbed pedestrians), we expect connected components with similar edge topology, weights, and annotation to exhibit similar dynamics and thus to be realizations of the same scenario. In this sense, we formally define a $S_{\text {sel }}$ by specifying an edge topology and ranges for weights and annotations. This selects a subgraph $\tilde{G}_{s} \subset \tilde{G}$, and all the connected components of $\tilde{G}_{s}$ are associated with the realization of the scenario.

We exploit this concept to retain data about avoidance dynamics of pairs (Sec. V). We find pairs of pedestrians in avoidance among the dyads (connected components of two nodes) in $\tilde{G}$. Specifically, we retain only those dyads in which (i) the walking directions of the two pedestrians are opposite and (ii) the two pedestrians initially faced each other. We call $\tilde{G}_{2, a}$ the subgraph of such dyads, to be used in the analyses in Sec. V. (As in the case of $\widetilde{G}_{1}$, also here we consider further technical constraints, discussed in Sec. III C.) In Fig. 5, we report a schematic description of the selection of avoidance pairs, while some examples of real data selected via the procedure reported here are in Fig. 6.

We finally stress that we use pairwise metric properties [i.e., $\vec{w}(e)$ ] as a discriminant of the occurrence of an interaction. It has been recognized recently that pedestrian interactions, especially at high densities, can be determined by factors beyond the sole metric [2] as it happens, e.g., for social animals [29]. As here we restrict the study to free-flow conditions and to one-to-one interactions, analyses based on metric arguments appear sufficient.

\section{Data sets}

In this technical section we discuss the restrictions and transformations further applied to the trajectory sets selected by $\tilde{G}_{1}$ and $\tilde{G}_{2, a}$ to yield the data sets employed in the following sections. The restrictions specified below identify further subgraphs within $\tilde{G}_{1}$ and $\tilde{G}_{2, a}$. We refrain however from introducing new symbols and maintain, with an abuse of notation, these identifiers for the subgraphs. 
TABLE II. Parameters employed in the construction of the graph-based representation (Sec. III), in the undisturbed dynamics model (Sec. IV), and in its extension to include pairwise avoidance (Sec. V). The pairwise avoidance model extends the undisturbed dynamics model and preserve all its parameters but the percentage of runners. This percentage is reduced following the observations.

\begin{tabular}{|c|c|c|}
\hline Parameter & Value & Note \\
\hline & & Graph-based representation \\
\hline$\overline{d_{m}}$ & $2.4 \mathrm{~m}$ & min distance for interaction \\
\hline$d_{y, m}$ & $0.8 \mathrm{~m}$ & $\begin{array}{l}\text { min transversal distance } \\
\text { for interaction }\end{array}$ \\
\hline$\tau_{m}$ & 5 frames $=1 / 3 \mathrm{~s}$ & min time for interaction \\
\hline \multirow[t]{2}{*}{$\tau_{M}$} & 20 frames $=4 / 3 \mathrm{~s}$ & $\begin{array}{l}\text { min interaction time } \\
\text { retained (Sec. III C) }\end{array}$ \\
\hline & & Undisturbed dynamics \\
\hline$\overline{u_{p, w}}$ & $1.29 \mathrm{~ms}^{-1}$ & walkers \\
\hline$u_{p, r}$ & $2.70 \mathrm{~ms}^{-1}$ & runners percentage \\
\hline$\sigma_{x}$ & $0.25 \mathrm{~ms}^{-3 / 2}$ & \\
\hline$\sigma_{y}$ & $0.25 \mathrm{~ms}^{-3 / 2}$ & \\
\hline$\alpha_{w}$ & $0.037 \mathrm{~m}^{-2} \mathrm{~s}$ & walkers \\
\hline$\alpha_{r}$ & $0.0015 \mathrm{~m}^{-2} \mathrm{~s}$ & runners percentage \\
\hline$\beta$ & $1.765 \mathrm{~m}^{-2} \mathrm{~s}$ & \\
\hline$v$ & $0.297 \mathrm{~s}^{-1}$ & \\
\hline & $0.0 \mathrm{~m}$ & \\
\hline \multirow[t]{2}{*}{ runners percentage } & $4.02 \%$ & \\
\hline & & Pairwise avoidance \\
\hline$\theta_{1}$ & $20^{\circ}$ & \\
\hline$\theta_{2}$ & $90^{\circ}$ & \\
\hline$\mu$ & $1.0 \mathrm{~s}^{-1}$ & \\
\hline$R$ & $2.4 \mathrm{~m}$ & \\
\hline$r$ & $0.6 \mathrm{~m}$ & \\
\hline$A$ & $1.5 \mathrm{~m} \mathrm{~s}^{-2}$ & \\
\hline$B$ & $0.7 \mathrm{~m} \mathrm{~s}^{-2}$ & \\
\hline runners percentage & $0.2 \%$ & \\
\hline
\end{tabular}

\section{Data set for diluted flow analysis (Sec. IV: constraints on $\tilde{G}_{\mathbf{1}}$ )}

(i) Restriction to straight intended paths and quasirectilinear trajectories. We aim at analyzing the fluctuations of the undisturbed motion when occurring around intended paths that are straight. In these conditions, we expect to observe trajectories that are quasirectilinear. From energy minimization arguments (cf. [30]), we expect intended paths to be straight when it comes to reach targets in obstacle-free environments. In our data set, we could however also observe largely erratic trajectories formed, e.g., of circular sections or of parabolic arcs. As these trajectories are out of our modeling purpose, we discard them after we identify them through the procedure described in Appendix B.

(ii) Coordinate system. Limiting our scope to quasirectilinear trajectories, we rotate them for convenience such that, in an $(x, y)$ reference system, $x$ is the longitudinal walking direction and $y$ is the direction of the transversal fluctuations (quasirectilinear trajectories have generally different inclinations depending on their starting position (cf. Fig. 14)). The details of the rotation procedure are in Appendix B.

\section{Data set for pairwise avoidance analysis}

(Sec. V: constraints on $\tilde{G}_{2, a}$ )

Time thresholding of pairwise dynamics. Due to the finiteness of our observation window, the joint observation time for a pairwise dynamics can be limited to a few frames. For instance, this occurs when a pedestrian of the pair is about to leave as the second enters the domain. To exclude these cases from our data set we impose a lower bound $\tau_{M}$ on the joint recorded time, i.e., we require $\tau>\tau_{M}$. We choose $\tau_{M}$ to be comparable to the crossing time of the observation window of an undisturbed pedestrian. Notably this restriction guarantees that the point in space at which the two pedestrians of the pair are closest is roughly in the middle of the observation window.

\section{UNDISTURBED MOTION}

In this section we model the dynamics of pedestrians walking undisturbed (also referred to as free flow), keeping as a quantitative reference the measurements collected from the setup in Fig. 2 (cf. Sec. II) and selected through $\tilde{G}_{1}$ (cf. Secs. III B and III C). The free flow motion is a limit condition for the dynamics, as it involves pedestrian densities at its lowest levels. We consider it as a reference condition for which we interpret pairwise interactions (analyzed in Sec. V) as perturbations. We proceed to deduce a model for undisturbed conditions and we then compare it with our measurements in terms of probability distribution functions.

Individuals crossing a large corridor typically move following straight intended paths along quasirectilinear trajectories (cf. Sec. III C). Besides this variability in the individual 


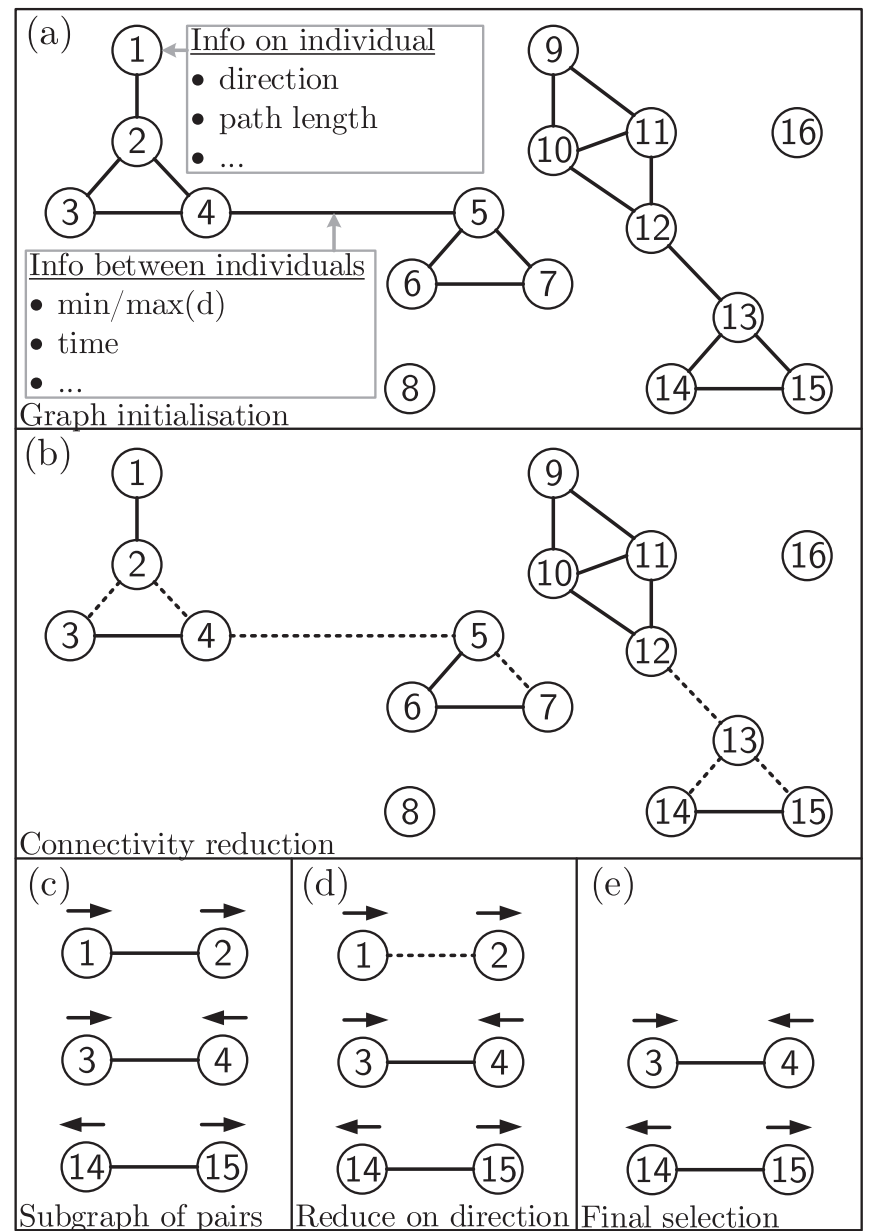

FIG. 5. Occurrences of mutual avoidance of two pedestrians walking in opposite directions are selected automatically using the graph representation. In this figure we summarize the whole process that leads from (a) $G$ to (e) the subgraph $\tilde{G}_{2, a}$. First, the graph representation in (a) is sparsified, removing the edge should two pedestrians not be interacting (according to the condition in Fig. 4), yielding the subgraph $\tilde{G}$ in (b). (c) We then isolate the subgraphs of $\tilde{G}$ constituted only of dyads (connected components with two nodes) that are isolated to further retain only (d) and (e) the cases in which the walking directions of pedestrians are opposite, i.e., one pedestrian walks toward the bus station and the other one toward the city center (cf. node annotations in Fig. 3). In this last step we further filter to retain pairs of pedestrians whose interaction time satisfies $\tau>\tau_{M}$.

intended paths, each pedestrian performs small and highfrequency random fluctuations (about $1 \mathrm{~Hz}$ due to walking physiology). Moreover, as observed in Ref. [12], rare large fluctuations in the motion occur too. Such rare large deviations include trajectory inversions. In Ref. [12], these two apparently independent fluctuating phenomena were treated as realizations of a unique Langevin stochastic dynamics with a bistable longitudinal velocity potential. In other words, the dynamics was treated in terms of a longitudinal velocity $u$ exhibiting small fluctuations around a stable state $u=u_{p}$ plus occasional velocity inversion events $u \rightarrow-u$ (for which the dynamics stabilizes on $u=-u_{p}$ ).

Here we provide a twofold extension of the Langevin model in Ref. [12] for the wider, longer, and less constrained

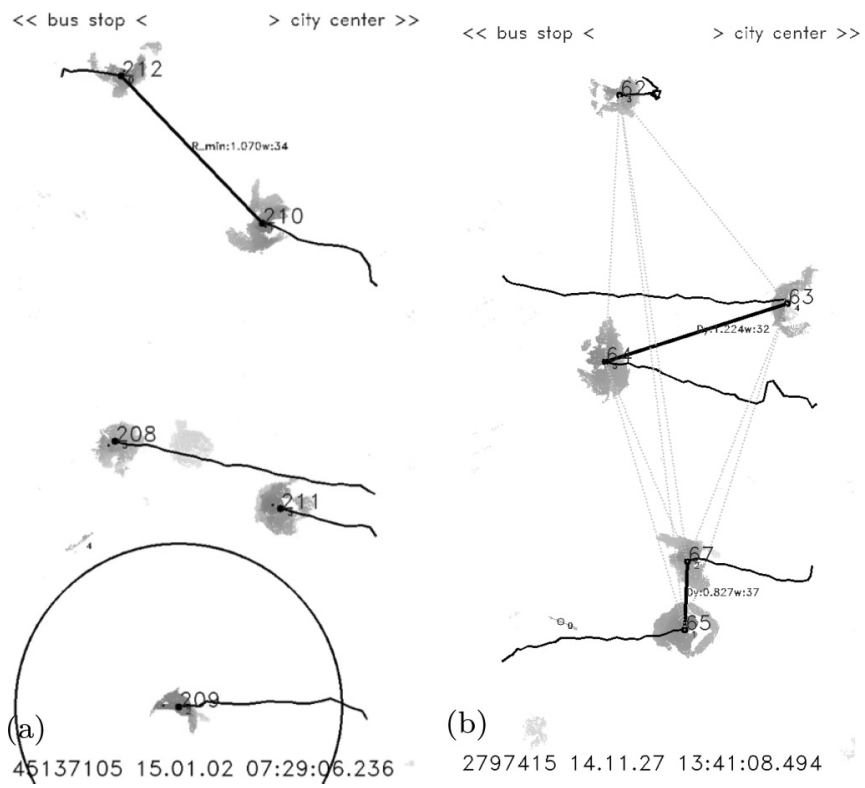

FIG. 6. Examples of depth maps collected in our experimental campaign (cf. Fig. 2 and Sec. II). Individual trajectories have been superimposed on the depth maps in postprocessing. Moreover, the panels include realizations of scenarios of interest. Panel (a) contains one realization of pairwise avoidance (pedestrians 210 and 212, joined by a thick black line) and one undisturbed pedestrian (209; we report the circular region of radius $d_{m}$ around them that remains not visited by others). Panel (b) contains two realizations of pairwise avoidance (pedestrians 63 and 64 and pedestrians 65 and 67). Note that we can have multiple pairwise avoidance realizations in the same frame, on the condition that there are no interactions between them. The graph representation flexibly allows us to define these scenarios and efficiently recover them in the measurements.

walking area considered (cf. Fig. 2). For the sake of completeness, we first present the extended model and then we discuss it in view of our previous work [12].

For convenience, we adopt a coordinate system $(x, y)$, where $x$ is a longitudinal coordinate along the walking direction, i.e., along the intended path, which we consider as a straight line parallel to the longitudinal direction of the corridor, and parametrized by the variable $y_{p}$. The variable $y$ accounts for transversal position, so $y-y_{p}$ identifies the fluctuation around the intended path. We model the longitudinal and transversal dynamics as uncorrelated Langevin motions satisfying

$$
\begin{aligned}
& \frac{d x}{d t}=u(t), \\
& \frac{d y}{d t}=v(t), \\
& \frac{d u}{d t}=f(u)+\sigma_{x} \dot{W}_{x}, \\
& \frac{d v}{d t}=-2 v v(t)+\beta g(y)+\sigma_{y} \dot{W}_{y},
\end{aligned}
$$

where $u$ and $v$ are, respectively, the longitudinal and transversal velocity components, $v$ and $\beta$ are positive model parameters, and $\dot{W}_{x}$ and $\dot{W}_{y}$ are independent, $\delta$-correlated-in-time Gaussian noise scaled by the positive coefficients $\sigma_{x}$ and $\sigma_{y}$. 


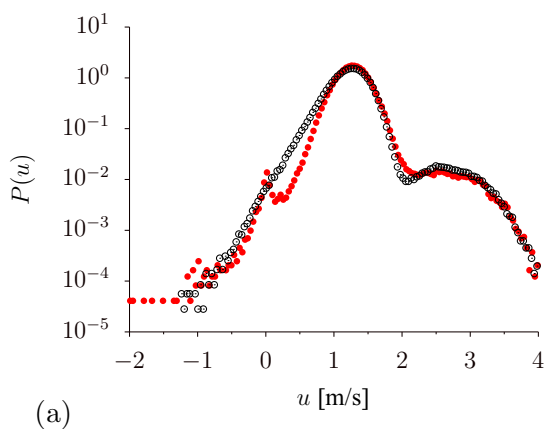

data $\quad$ simulations
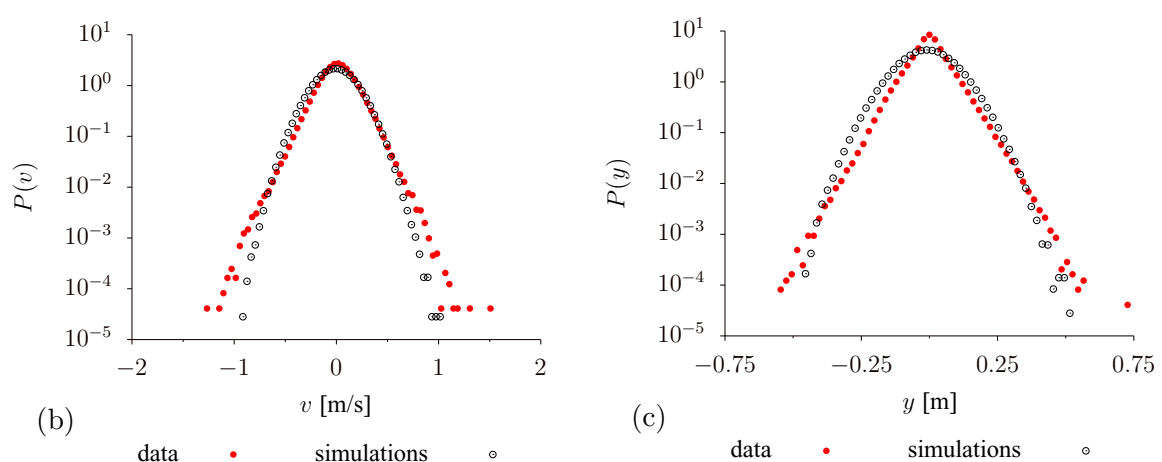

FIG. 7. Probability distribution functions of walking velocity and positions for undisturbed individuals: comparison between measurements (filled red dots) and simulations of Eqs. (4)-(7) (empty black circles) (simulation parameters listed in Table II). The panels contain (a) longitudinal velocities $u$, (b) transversal velocities $v$, and (c) transversal positions ( $y$, assuming $y_{p}=0$ ). Pedestrians walk most frequently at around $1.29 \mathrm{~m} / \mathrm{s}$ [cf. (a)]. In addition, we observe a tiny fraction of running pedestrians, about $4 \%$, contributing to the hump at above $2 \mathrm{~m} / \mathrm{s}$ and pedestrians turning back, providing negative velocities contributions. (b) Transversal fluctuations in velocity appear well approximated by a Gaussian distribution, while (c) transversal positions exhibit small deviations from a Gaussian behavior. Our model [Eqs. (4) and (6)] reproduces quantitatively the complete longitudinal velocity statistics inclusive of the running hump as well as the inversion events. The transversal dynamics is also well approximated as a stochastic damped harmonic oscillator [Eqs. (5) and (7)].

(We assume, consistently with [12], $\sigma_{x}=\sigma_{y}$; cf. Table II. Our choice for the noise is common and made for simplicity, yet it is not mandatory; see, e.g., [5].) The features of the dynamics are finally incorporated in the two functions $f(u)$ and $g(y)$.

As in Ref. [12], we choose $f(u)$ as possibly the simplest smooth model for a bistable dynamics, i.e., as the gradient of a double-well (velocity) potential. In formulas it reads

$$
f(u)=-4 \alpha_{i} u\left(u^{2}-u_{p, i}^{2}\right)=-\partial_{u} \alpha_{i}\left(u^{2}-u_{p, i}^{2}\right)^{2},
$$

with $\pm u_{p, i}$ the expected stable velocities and $\alpha_{i}$ the modulating factor of the force. As a first extension of the model in Ref. [12], we introduce the subscript $i$ to enable multiple populations all behaving identically except for the stable velocity value. This allows one to distinguish, e.g., people walking at usual speed and runners.

The function $g(y)$ models the restoring impulse towards the intended path. In formulas, it reads

$$
g(y)=-2\left(y-y_{p}\right)=-\partial_{y}\left(y-y_{p}\right)^{2} .
$$

This marks a second, yet fundamental, extension to the model in Ref. [12]. In fact, a wide corridor enables a continuous choice of straight intended paths that remain unchanged during the motion (in formulas, $\dot{y}_{p}=0$ ). In turn, in Sec. V we describe interactions considering a dynamics also for the variable $y_{p}$.

In Fig. 7 we compare the measured and modeled pedestrian motion in terms of probability distribution functions of longitudinal and transversal velocity and transversal fluctuations with respect to the intended path. The figures include data from $N=47122$ trajectories of average time length $2 \mathrm{~s}$ (i.e., 31 frames). Approximately $34 \%$ of the trajectories are from undisturbed pedestrians walking towards the bus terminal (the rest are from undisturbed pedestrians walking towards the city center). The comparison is performed with 47122 trajectories simulated via (4)-(7) and calibrating the parameters as in Ref. [12] (values reported in Table II).

In the longitudinal velocity $(u)$ probability distribution [Fig. 7(a)] we observe different regimes: Most likely people walk with speed fluctuating around $1.29 \mathrm{~m} / \mathrm{s}$. Moreover, about $4 \%$ of the pedestrians run across the walkway: This results in the hump on the right-hand side of the distribution. Finally, rare events such as turning back trajectories and stopping are present which provide contributions in the left tail and at around $0 \mathrm{~m} / \mathrm{s}$, respectively. By adopting the measured ratio of walkers and runners, simulations quantitatively reproduce the observed velocity distribution. We observe Gaussian transversal fluctuations of the velocity ( $v$ ) that Eqs. (5) and (7) capture. Slight deviations from the predicted Gaussian fluctuations in transversal position $(y)$ are instead observed.

\section{PAIRWISE AVOIDANCE}

In this section we model the dynamics of the pairwise avoidance and of the related changes in the intended path (cf. the conceptual sketch in Fig. 1 and measured cases in Fig. 6). We consider these dynamics in the simplest condition involving exclusively two pedestrians walking in opposite directions and avoiding each other while remaining sufficiently far (i.e., not influenced) from any other individuals. In this sense, we deal with avoidance in diluted conditions. We compare with our measurements selected through the (connected components of the) subgraph $\tilde{G}_{2, a}$ (cf. Secs. III B and III C; each of the two nodes of the dyads in $\tilde{G}_{2, a}$ corresponds to one of the two pedestrians involved). This scenario represents for us the first building block to treat quantitatively interaction dynamics on top of the undisturbed motion (Sec. IV). In the following we first describe our model and then we compare it with the measurements in terms of probability distributions.

To model the pairwise avoidance we consider two individuals, each modeled following Eqs. (4)-(7) plus coupling terms affecting both individual positions $(x, y)$ and individual planned paths $y_{p}$. In other words, the individual state is now described by the triplet $\left(x, y, y_{p}\right)$ (and derivatives), with $y_{p}$ entering the dynamics as a variable and not as a constant parameter. We model the coupling terms as social forces [10,31], acting on the whole triple $\left(x, y, y_{p}\right)$ (Fig. 8). 


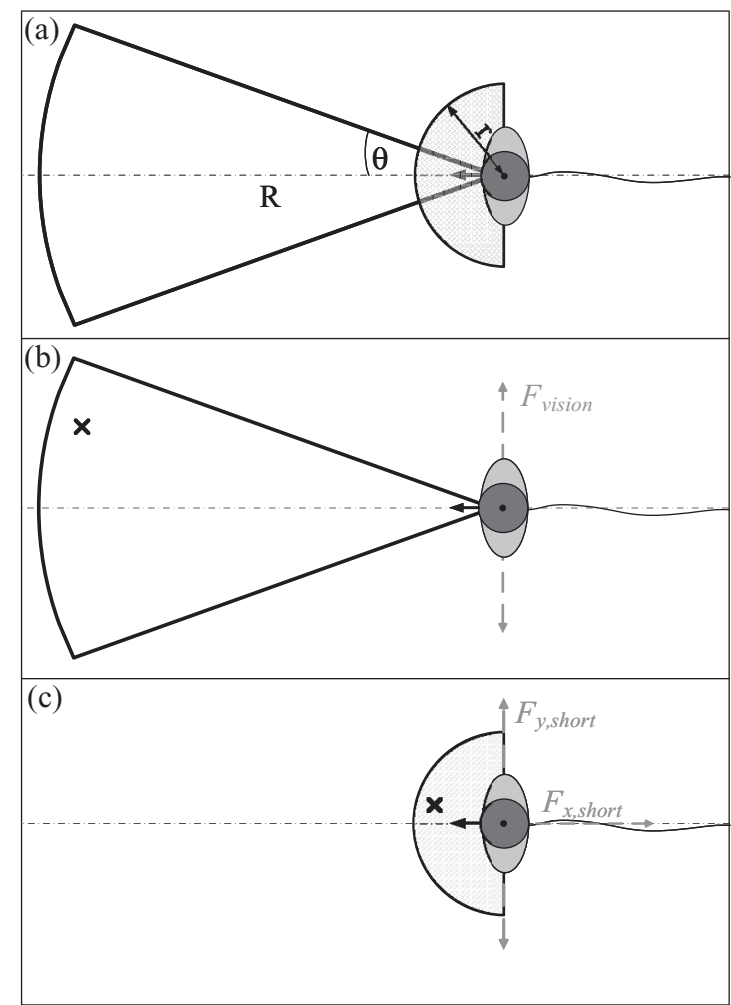

FIG. 8. Schematics of the interaction forces considered. (a) The intended path of a pedestrian is modified on a twofold basis: by long-range, sight-based (thus anisotropic) forces [cf. Eq. (16)] and by short-range contact-avoidance forces [cf. Eq. (17)]. Long-range forces are bounded within a circular sector of radius $R$ and angular semiamplitude $\theta$ located in front of the individual (i.e., aligned with the intended path). Short-range forces are frontal and bounded within a circular region of radius $r$. (b) Long-range sight-based interactions, e.g., with the pedestrian marked with a cross, yield forcing $F_{\text {vision }}$ in the orthogonal direction with respect to the intended path. (c) Short-range forces provide isotropic $F_{\text {short,x }}=F_{\text {short, } \mathrm{y}}$, although frontal only, contact avoidance, e.g., of the pedestrian marked with a cross.

Let $p_{1}$ and $p_{2}$ be two pedestrians in an avoidance event. Adopting the point of view of one of the two, say, $p_{1}$, and using the same $(x, y)$ reference system used in Sec. IV, we model the dynamics as

$$
\begin{aligned}
& \frac{d y_{p}}{d t}=\dot{y}_{p}(t) \\
& \frac{d \dot{y}_{p}}{d t}=F_{\mathrm{vision}}-2 \mu \dot{y}_{p}(t) \\
& \frac{d x}{d t}=u(t) \\
& \frac{d y}{d t}=v(t) \\
& \frac{d u}{d t}=-4 \alpha_{i} u\left(u^{2}-u_{p, i}^{2}\right)+\sigma_{x} \dot{W}_{x}-e_{x} F_{\text {short }} \\
& \frac{d v}{d t}=-2 v v-2 \beta\left(y-y_{p}\right)+\sigma_{y} \dot{W}_{y}-e_{y} F_{\text {short }}+F_{\text {vision }}
\end{aligned}
$$

where the unit vector of components $\left(e_{x}, e_{y}\right)$ is directed from $p_{1}$ to $p_{2}$ and $\mu$ is a positive model parameter. We superimpose on the undisturbed dynamics in Eqs. (4)-(7) two (social) forces $F_{\text {short }}$ and $F_{\text {vision, encompassing respec- }}$ tively, two influencing elements of the interaction dynamics (see also sketches in Fig. 8). Here $F_{\text {short }}$ is a shortranged contact-avoidance force; it mimics one's immediate and strong collision-avoidance reaction to individuals in the very vicinity and acts on the velocity variables $u$ and $v$. In turn, $F_{\text {vision }}$ mimics the sight-based avoidance maneuvers having longer and anisotropic range. In addition, $F_{\text {vision }}$ acts in the transversal direction only, affecting both, and equally, the transversal velocity $v$ and the intended path $y_{p}$. This modeling choice follows the idea confirmed from measurements that avoidance not only yields lateral motion, but also provides a persistent change of our intended paths.

We model both forces with a decaying exponential of the squared distance between pedestrians (as common practice in the pedestrian dynamics community [10]). In formulas, they read

$$
\begin{aligned}
F_{\text {vision }} & =-\operatorname{sgn}\left(e_{y}\right) A \exp \left(-d^{2} / R^{2}\right) \chi_{1}(\tilde{\theta}), \\
F_{\text {short }} & =B \exp \left(-d^{2} / r^{2}\right) \chi_{2}(\tilde{\theta}),
\end{aligned}
$$

where $A$ and $B$ are positive parameters, $d$ is the (scalar) distance between the two considered pedestrians (cf. Fig. 1), $r$ and $R$ are scaling factors for the interaction ranges, $\tilde{\theta}$ is the angle between the line joining the two pedestrians and the horizontal, and $\chi_{j}(\tilde{\theta})=1$ for $|\tilde{\theta}|<\theta_{j}$ and 0 otherwise $(j=1,2)$.

The coupled systems of Langevin equations (10)-(15) for $p_{1}$ and $p_{2}$ feature, as a whole, a one-dimensional translational symmetry group. Letting $\left(x_{1}, y_{1}, y_{p, 1}\right)$ and $\left(x_{2}, y_{2}, y_{p, 2}\right)$ be the states of $p_{1}$ and $p_{2}$, respectively, the symmetry reads

$$
y_{1}, y_{p, 1}, y_{2}, y_{p, 2} \rightarrow y_{1}+c, y_{p, 1}+c, y_{2}+c, y_{p, 2}+c
$$

for any real number $c$. In other words, the dynamics is invariant up to a rigid translation of the transversal position and planned path of both individuals.

We compare the model and data first in terms of the absolute transversal distance between $p_{1}$ and $p_{2}:|\Delta y|=\left|y_{1}-y_{2}\right|$ (cf. Fig. 9) (Similarly to the undisturbed case discussed in Appendix B, pairs of measured trajectories underwent also a rotation procedure to align with our coordinate system. Details are reported in Appendix C.) We expect $|\Delta y|$ to well approximate the transversal distance between the intended paths $\left|y_{p, 1}-y_{p, 2}\right|$ once considered at the ensemble level, i.e.,

$$
\langle\Delta y\rangle_{\mathrm{ens}}=\left\langle\left|y_{1}-y_{2}\right|\right\rangle_{\mathrm{ens}} \approx\left\langle\left|y_{p, 1}-y_{p, 2}\right|\right\rangle_{\mathrm{ens}},
$$

where $\langle\cdot\rangle_{\text {ens }}$ denotes an ensemble average over the measurements (we indicate with $\mathbb{E}_{\text {ens }}[\cdot \mid E]$, the conditioned ensemble average, where $E$ is the conditioning event). We expect, in fact, individual fluctuations with respect to the intended paths be negligible after the (ensemble) average.

We measure $|\Delta y|$ at three phases of the interaction: (i) the first appearance of $p_{1}$ and $p_{2}$ in our observation window $\left[\left|\Delta y_{i}\right|\right.$ in Fig. 9(a)], (ii) the time instant, in the following referred to as $t_{s}$, when the pair is side-by-side $\left[\left|\Delta y_{s}\right|\right.$ in Fig. 9(b)], and (iii) the last simultaneous appearance of the pair in our observation window $\left[\left|\Delta y_{e}\right|\right.$ in Fig. 9(c)]. We report our measurements and simulation results in terms of two 


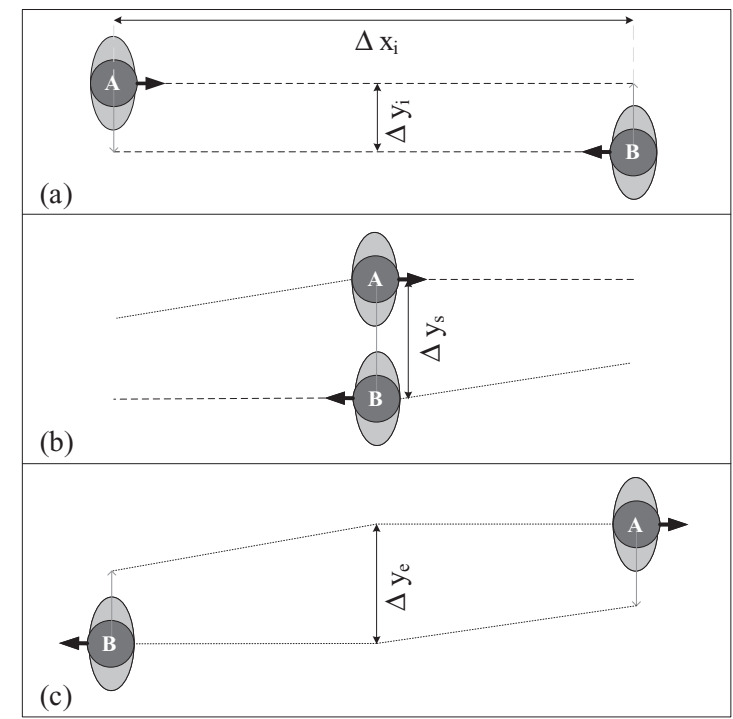

FIG. 9. Considered phases of the counterflowing pairwise dynamics for the scatter plots in Figs. 10 and 11. (a) Sketch of the entrance, i.e., the first moment of simultaneous appearance of the pedestrian pair in our observation window. (b) Sketch of side-byside walking, which occurs when the two pedestrians have the same longitudinal position. (c) Sketch of the exit, i.e., the last moment in which the two pedestrians appear together. (a) $\left|\Delta y_{i}\right|$, (b) $\left|\Delta y_{s}\right|$, and (c) $\left|\Delta y_{e}\right|$ indicate the absolute lateral distance between the two pedestrians in phases (a), (b), and (c), respectively (see also (i)-(iii) in Fig. 1).

scatter plots targeting two halves of the interaction dynamics: $\mathrm{S} 1$, describing the avoidance maneuvers until the side-by-side phase [plane $\left(\left|\Delta y_{i}\right|,\left|\Delta y_{s}\right|\right)$, Fig. 10], and S2, describing the following regime [plane $\left(\left|\Delta y_{s}\right|,\left|\Delta y_{e}\right|\right)$, Fig. 11].

For S1 (and analogously for S2), a synthetic view of the data can be obtained by computing the ensemble average of $\left|\Delta y_{s}\right|$ conditioned to $\left|\Delta y_{i}\right|$, namely,

$$
e\left(\left|\Delta y_{s}\right|\right)=\mathbb{E}_{\mathrm{ens}}\left[\left|\Delta y_{s}\right||| \Delta y_{i} \mid\right] .
$$

Considering the approximation in Eq. (18), we expect this function to represent the deviation of the intended paths. The scatter plots in Figs. 10-12 include data from 9089 avoidance events (i.e., pairs of pedestrians) either experimentally measured (left panels) or simulated (right panels). In Figs. 12(a) and 12(b) we compare data and simulations in terms of the average conditioned distances [Eq. (19)] for cases S1 and S2, respectively. We observe the following.

Case $S 1$. We expect avoidance maneuvers, especially when collision is imminent, i.e., for $\left|\Delta y_{i}\right| \lesssim s_{b}, s_{b}$ being the size scale of the human body. In this condition, we expect a modification of intended paths to yield $\left.\left\langle\left|\Delta y_{s}\right|\right\rangle_{\mathrm{ens}}\right\rangle\left\langle\left|\Delta y_{i}\right|\right\rangle_{\mathrm{ens}}$, which is consistent with our measurements in Fig. 10(a). From the experimental measurements we have

$$
\left\langle\left|\Delta y_{s}\right|\right\rangle_{\mathrm{ens}} \approx 0.75 \mathrm{~m} \text { for }\left|\Delta y_{i}\right| \rightarrow 0
$$

and similarly in the case of simulations [Fig. 10(b)]. In contrast, a decreasing influence of $\left|\Delta y_{i}\right|$ on $\left|\Delta y_{s}\right|$ is expected as the former increases, since no interaction is at play at large transversal distances. As a consequence, we expect the average trend

$$
\left\langle\left|\Delta y_{s}\right|\right\rangle_{\mathrm{ens}} \approx\left\langle\left|\Delta y_{i}\right|\right\rangle_{\mathrm{ens}} \text { for }\left|\Delta y_{i}\right| \gg s_{b},
$$

i.e., a relaxation of $\left\langle\left|\Delta y_{s}\right|\right\rangle_{\text {ens }}$ towards the diagonal of the plane $\left(\left|\Delta y_{i}\right|,\left|\Delta y_{s}\right|\right)$. We observe such an expected trend [which we obtain in simulations per the scaling and anisotropy in Eq. (16)] only for $\left|\Delta y_{i}\right|<1.4 \mathrm{~m}$. In the case $\left|\Delta y_{i}\right|>$ $1.4 \mathrm{~m},\left\langle\left|\Delta y_{s}\right|\right\rangle_{\text {ens }}$ lies slightly below the diagonal line, suggesting an average end-distance contraction. As the distance increases, false positive and false negative cases emerge in the selection operated by $\tilde{G}_{2, a}$. In this case, these determine the overweight of the region below the diagonal in Fig. 10(a), thus the inflection of the $\left\langle\left|\Delta y_{s}\right|\right\rangle_{\text {ens }}$ curve.

Case S2. The interaction dynamics in Fig. 1 conjectures that the change in intended path mostly occurs to ensure avoidance, hence before the two pedestrians are closest in space. Afterward it plays a negligible role. In formulas the conjecture reads

$$
y_{p, 1}-y_{p, 2} \approx \text { const } \quad \text { (postinteraction). }
$$

Considering the approximation in Eq. (18), Eq. (22) is consistent with our measurements and simulations, reported in Figs. 11(a) and 11(b), respectively. In these,

$$
\left\langle\left|\Delta y_{e}\right|\right\rangle_{\mathrm{ens}} \approx\left\langle\left|\Delta y_{s}\right|\right\rangle_{\mathrm{ens}} \quad \text { (postinteraction) }
$$

holds for $\left|\Delta y_{s}\right|>0.8 \mathrm{~m}$, while at lower $\left|\Delta y_{s}\right|$, i.e., for very close passing distances (observed very rarely), we measure a small tendency to increase the transversal distance in the postinteraction stage. This can be an inertial phenomenon: Pedestrians avoiding each other, yet passing by each other at very close distance, keep on increasing their mutual distance. This aspect is modeled via the frontal isotropic short-range contact-avoidance force in Eq. (17).

Avoidance impacts further on the walking speed $s=$ $\sqrt{u^{2}+v^{2}}$, which we adopt as a second comparison term between model and experimental measurements. Around the time instance $t_{s}$ of minimum distance [Fig. 9(b)] speed is temporarily adjusted and reduced from the undisturbed flow regime (Fig. 7). Considering a time window spanning $0.66 \mathrm{~s}$ before $t_{s}$ (i.e., $\left[t_{s}-0.66 \mathrm{~s}, t_{s}\right]$ ) and a time window spanning $0.66 \mathrm{~s}$ after $t_{s}$ (i.e., $\left[t_{s}, t_{s}+0.66 \mathrm{~s}\right]$ ), in Figs. 13(a) and 13(b), respectively, we compare the speed distributions measured and predicted by the model. Also in this case we find excellent agreement.

Pairwise avoidance, finally, is not always a successful operation. As a rare event, two people can bump into each other, having their minimum distance $\min (d)$ becoming comparable to their diameter. It is important that such rare events remain captured, in statistical terms, by the model, e.g., for their implication in safety. In Fig. 13(c) we report the cumulative distribution of collisions as a function of $\min (d)$. In the range $[0.3,0.6] \mathrm{m}$ the distribution measured and the distribution predicted by the model present an exponential growth in perfect agreement, with about 40 cases recorded in both cases for $\min (d) \leqslant 0.5 \mathrm{~m}$.

\section{DISCUSSION}

In this paper we investigated quantitatively the pedestrianpedestrian avoidance interactions occurring in diluted 

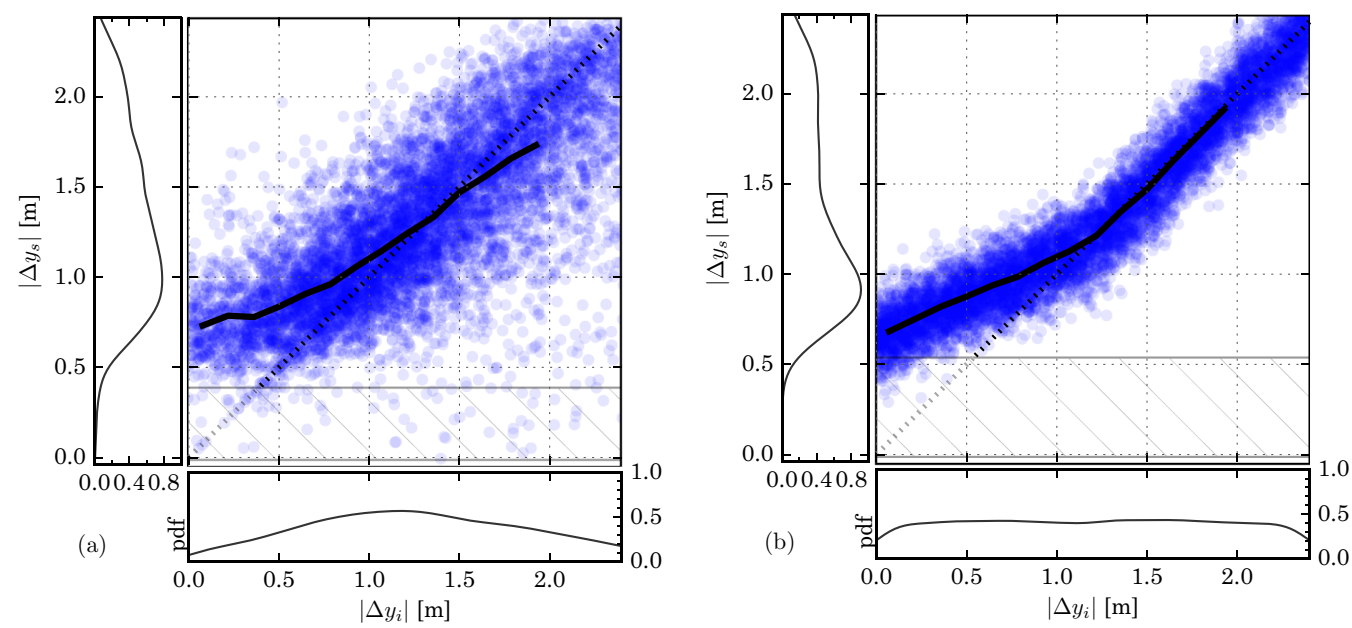

FIG. 10. Absolute lateral distance at the entrance [ $\left|\Delta y_{i}\right|, x$ axis; cf. scenario (a) in Fig. 9] vs absolute lateral distance when side-by-side $\left[\left|\Delta y_{s}\right|, y\right.$ axis; cf. scenario (b) in Fig. 9]. Each sample in the scatter plot represents (a) a measured or (b) a simulated pair of pedestrians in counterflow. The function $e\left(\left|\Delta y_{s}\right|\right)$ is reported as a solid line [cf. the definition in Eq. (19), i.e., $e\left(\left|\Delta y_{s}\right|\right)$ encodes the ensemble average value of $\left|\Delta y_{s}\right|$ conditioned on $\left.\left|\Delta y_{i}\right|\right]$. The diagonal $\left|\Delta y_{s}\right|=\left|\Delta y_{i}\right|$ represents the dynamics for which the lateral distance of a pedestrian pair remains unchanged between scenarios (a) and (b) (in formulas $\frac{d \Delta y}{d t}=0$ ). In other words, both pedestrians walked straight (modulo a constant lateral offset). $e\left(\left|\Delta y_{s}\right|\right)$ departs from the diagonal for low $\left|\Delta y_{i}\right|$, which identifies pedestrians walking towards each other, and avoiding each other. As $\left|\Delta y_{i}\right|$ grows, interactions and collisions vanish, hence the asymptotic tendency toward the diagonal line. A synthetic comparison limited to the average trends [Eq. (18)] is reported in Fig. 12(a). The bottom and left panels in (a) and (b) report the (marginal) distributions of $\left|\Delta y_{i}\right|$ and $\left|\Delta y_{s}\right|$.

conditions comparing with measurements obtained in a reallife pedestrian tracking campaign. As two individuals walk in opposite directions on a shared space, avoidance maneuvers become necessary should a collision be avoided; these maneuvers affect, at the same time, the path observed and the intended path. We modeled this scenario in terms of a sight-dependent interaction force and a collision-deterring force, which we superimposed on a Langevin model for the undisturbed pedestrian motion. Overall the state of each individual was treated as a triple of variables including the components of the position plus a spatial variable representing the intended path.

We performed the experimental campaign employing a state-of-the-art pedestrian tracking method, which in a period of over 6 months enabled us to collect a data set of about five millions high-resolution individual trajectories. Using real-life data acquired from 24/7 tracking allows us, at the same time, to accurately quantify characteristic fluctuations and rare events in the dynamics (as events appearing only once in 1000 or once in 10000 cases can be measured)
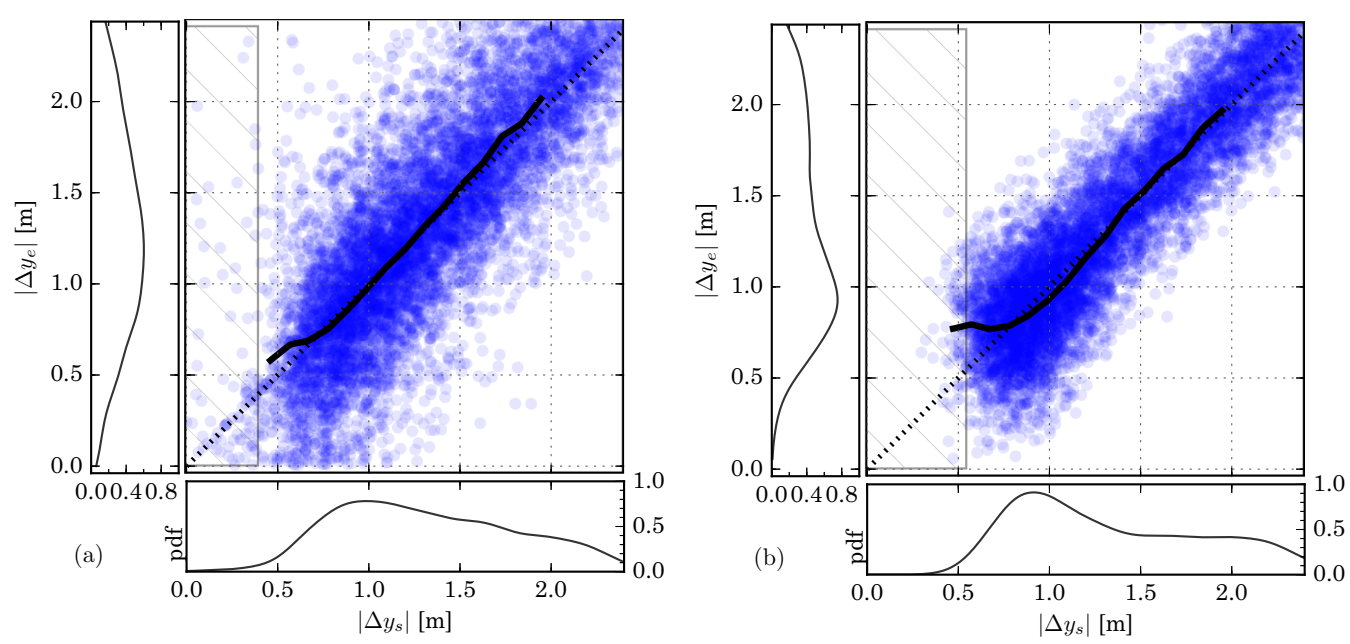

FIG. 11. Absolute lateral distance when side-by-side $\left[\left|\Delta y_{s}\right|, x\right.$ axis; cf. scenario (b) in Fig. 9] vs absolute lateral distance at the exit [| $\Delta y_{e} \mid$, $y$ axis; cf. scenario (c) in Fig. 9]. Each sample in the scatter plot represents (a) a measured or (b) a simulated pair of pedestrians in counterflow. Once avoidance is ensured, the lateral distance is maintained until the exit from our observation window, as the scatter sample is concentrated around the diagonal $\left|\Delta y_{s}\right|=\left|\Delta y_{e}\right|$. A synthetic comparison including only the average trends [Eq. (18)] is reported in Fig. 12(b). 

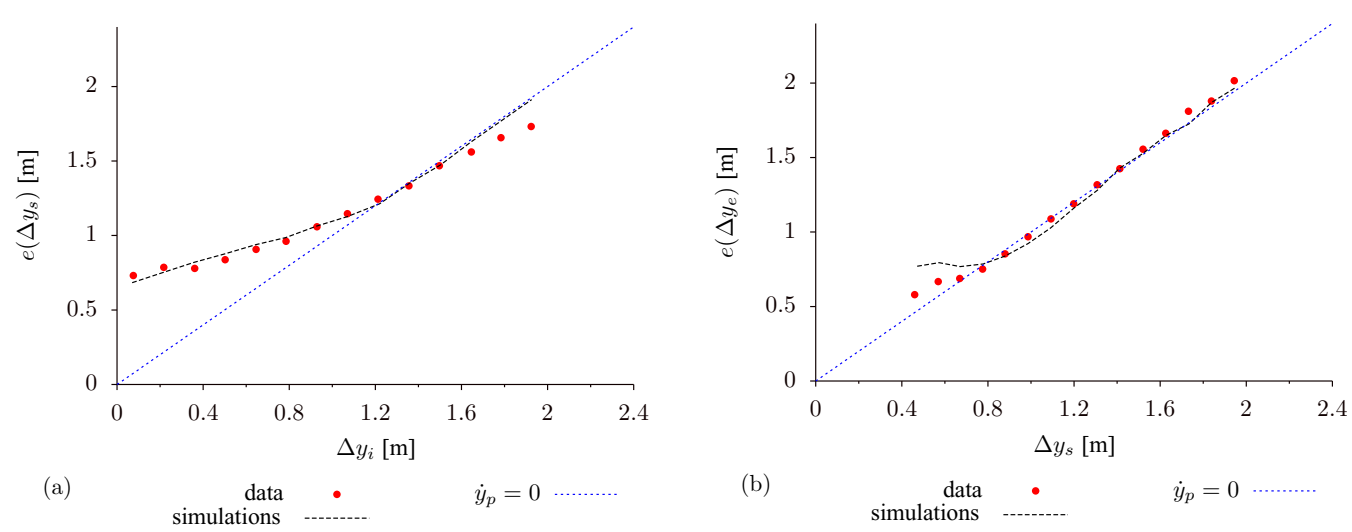

FIG. 12. Average absolute lateral distance: comparison between data and simulations. (a) Absolute lateral distance when at the entrance $\left(\left|\Delta y_{i}\right|, x\right.$ axis) vs side-by-side [conditioned average $e\left(\left|\Delta y_{s}\right|\right), y$ axis]. Data and simulation lines are reported from Figs. 10(a) and 10(b), respectively. (b) Absolute lateral distance when side-by-side $\left(\left|\Delta y_{s}\right|, x\right.$ axis) vs at the exit [conditioned average $e\left(\left|\Delta y_{e}\right|\right), y$ axis]. Data and simulation lines are reported from Figs. 11(a) and 11(b), respectively.

and avoid potential biases related, e.g., to the construction of laboratory or artificial experimental conditions (in which the dynamics to measure has been predefined and enforced by the experimenter). Acquiring data in a real-life scenario, however, is somehow similar to acquiring data from different laboratory experiments, each having different experimental parameters, that follow one another in random order. Ideally one wants to retain only the measurements pertaining to the occurrences of a selected scenario and aggregate them to perform ensemble statistical analyses. As operating a manual selection of measurements (as done in the past) would be impossible at the scale of our data set, in the first half of the paper we proposed a method efficiently automatizing the selection. Representing the measurement through a graph, we were able to formally define scenarios of interest as well as efficiently identify them within the data set. Selecting pairwise avoidance events in diluted conditions implies, from a naive perspective, a nonlinear scan of the data set: We are searching for pairs of trajectories that are mutually close while being far from any other at any time. With the method proposed, a single pass of the data set first and a linear pass of the graph edges next are sufficient to identify all the target events.

We analyzed the dynamics considering probability distribution functions that, due to our extensive data set, result in being very well resolved even in the tails (rare events). Our models target and reproduce quantitatively the stochastic behavior observed. At the level of the undisturbed motion, considering a mixture of walkers and runners, we could reproduce the nontrivial longitudinal dynamics which shows fluctuations around two average speed values, one for walkers and one for runners, plus rare U-turning events. This was possible via considering a double-well, i.e., bistable, velocity potential for each of the two populations. The interplay of the white noise excitations and the gradient-type velocity dynamics captures both small fluctuations around the average velocity and rare velocity inversions, which occur with a transition to the negative velocity stable state.

We addressed pairwise avoidance considering social-forcelike interactions between undisturbed pedestrians, which we extended to affect a hidden variable of the system introduced
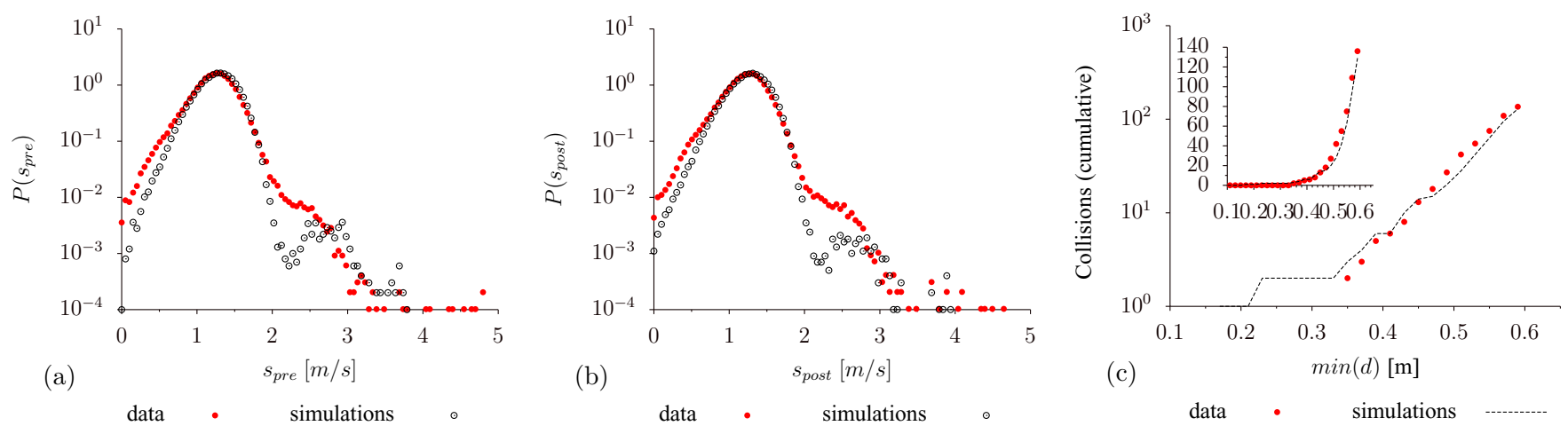

FIG. 13. Speed distribution (i.e., distribution of the velocity modulus $s$ ) (a) before and (b) after the encounter. Each time frame contributes one measurement per pedestrian. (a) Frames in the time interval $\left[t_{s}-0.66 \mathrm{~s}, t_{s}\right]$, where $t_{s}$ is the time instant of the side-by-side encounter, are considered. (b) The considered time interval is $\left[t_{s}, t_{s}+0.66 \mathrm{~s}\right]$. Note that for the pedestrians that are interacting, we have a reduced number of runners $(0.2 \%)$, and this is shown in the smaller percentage of speed values that are larger than $2 \mathrm{~m} / \mathrm{s}$. (c) Cumulative distribution of the minimum distance $\min (d)$ between the two pedestrians of the pair. Data and simulation are compared; the inset shows the same distribution on a linear scale. This cumulative distribution expresses the number of collisions that occurred vs predicted. Effectively a collision happens for $\min (d)$ smaller than or equal to the body diameter (about $0.5 \mathrm{~m}$ ). Experimental data and simulations agree quantitatively on the exponential decaying trend. 
in this work: the individual intended path. Despite being hidden for single realizations, we believe that the variations in intended path can be measured on the basis of ensemble averages, which we computed on the transversal distances between the pedestrians. Should pedestrians be in the path of a possible collision (initial transversal distance below $1.4 \mathrm{~m}$ ), their intended paths are deflected, such that when passing by one another the mutual distance is no lower than $0.75 \mathrm{~m}$. After the moment the passing occurs, no further modifications in intended paths were recorded. By including the intended path in the dynamics and subjecting it to the social force, we could reproduce quantitatively the observed dynamics including speed reductions in the proximity of the passing as well as the number of collision events.

This work is a methodological and modeling step to treat quantitatively, in a statistically accurate sense, interactions in crowd dynamics. We believe that the present approach can be extended to analyze situations characterized by higher complexity and density, increasingly common in civil infrastructures.

\section{ACKNOWLEDGMENTS}

We acknowledge the Brilliant Streets research program of the Intelligent Lighting Institute at the Eindhoven University of Technology, Nederlandse Spoorwegen, and the technical support of A. Muntean, T. Kanters, A. Holten, G. Oerlemans and M. Speldenbrink. During the development of the infrastructure, A.C. has been partly funded by a Lagrange Ph.D. scholarship granted by the CRT Foundation, Turin, Italy and by the Eindhoven University of Technology, the Netherlands. This work is part of the JSTP research programme "Vision driven visitor behaviour analysis and crowd management" with NWO Project No. 341-10-001, which is financed by the Netherlands Organisation for Scientific Research (NWO). Support from COST Action MP1305 "Flowing Matter" is also kindly acknowledged.

\section{APPENDIX A: TECHNICALITIES OF PEDESTRIAN TRACKING AT EINDHOVEN TRAIN STATION}

Our data collection employed an array of four overhead Kinect $^{\mathrm{TM}}$ sensors in order to obtain our depth-map streams at VGA resolution $(640 \times 480$ pixels $)$ and at 15 frames/s $(\mathrm{fps})$. The four views were in partial overlap and were merged into one large canvas like those in Fig. 6. The merging algorithm and registration algorithms, treated in Ref. [14], rely on the fact that a depth map is an overhead perspective view containing (by definition) the height of each pixel. Such information is sufficient to obtain an axonometric view (i.e., an aerial view in the limit of a far observer) from each of the four streams. Considering a depth map via the cylindrical coordinates $(\theta, \rho, h)$, where $\rho$ is a radial coordinate (i.e., $\rho=0$ is the image central axis), $\theta$ is an angle spanning the image space around the image axis, and $h$ is the altitude from the ground (normalized such that $h=1$ is the floor and $h=0$ is the camera plane), we employed the mapping

$$
(\theta, \rho, h) \mapsto(\theta, \rho h, h)
$$

that displaces each point to its vertical line. Note that $\rho h \leqslant$ $\rho$ holds and $\rho h=\rho$ is true only at the ground level (i.e., the ground level is invariant under this transformation). Extracting the lowest depth value (topmost) for each vertical line yields the desired axonometric view. Axonometric views can be merged by simple superposition which requires just the knowledge of the reciprocal positions of the cameras. Such registration and calibration steps have been performed manually after sliding a cart endowed with elements of known size underneath the cameras (cf. [14] and the Appendix in [32]).

The four Kinect ${ }^{\mathrm{TM}}$ sensors were connected in pairs to two computers whose system time has been synchronized with $O(1)$ ms precision through Network Time Protocol (NTP). To the best of our knowledge, the moment at which a depth image is taken by a Kinect ${ }^{\mathrm{TM}}$ is not controllable or triggerable. Hence, we let the four sensors record (maintaining $15 \mathrm{fps}$ ) and then we associated simultaneous images a posteriori. This yields a maximum error of $33 \mathrm{~ms}$, i.e., approximately $3 \mathrm{~cm}$ considering an average walking speed of $1 \mathrm{~m} / \mathrm{s}$, i.e., less than 7 pixels (where the conversion 230 pixel $\approx 1$ m holds).

Merged depth images were processed to detect pedestrian positions via the stochastic clustering algorithm proposed independently in Refs. $[25,26]$. We employed the same implementation of our previous works [12,23,33], to which we refer for further details.

Differently from $[12,23,33]$, we revised the tracking approach for increased robustness. As in Refs. [12,23,33], pedestrians are tracked by employing the classical particle tracking velocimetry (PTV) approach (e.g., [34]) and specifically through the OpenPTV library [35]. However, each detected pedestrian is considered via five different points: (i) the centroid of the body, (ii) the estimated head top position (centroid of the points within the fifth depth percentile), (iii) the estimated head-neck position (centroid of the points within the tenth depth percentile), (iv) the estimated head-neck-torso centroid (centroid of the points within the 20th depth percentile), and (v) the upper half of the body (centroid of the points within the 50th depth percentile).

We performed five independent trackings, considering only one of these five positions at a time and for all pedestrians. Hence, we compared the five tracking results for consistency, considering reliable the tracks for which at least three out of five tracking results were in agreement.

We smoothed the obtained trajectories for noise reduction with the Savisky-Golay algorithm [36], a common approach in PTV (e.g., [37]), with window size 5 and polynomial degree 2.

\section{APPENDIX B: COORDINATE TRANSFORMATION FOR UNDISTURBED PEDESTRIAN TRAJECTORIES}

In Fig. 14 we include different average measurements of the undisturbed motion. We observe that pedestrian trajectories, although generally straight, are not parallel to the axis of the station walkway (i.e., the horizontal direction in Fig. 14). To employ the $(x, y)$ coordinate system introduced in Sec. IV, we rotate each trajectory to have the longitudinal direction aligned with the $x$ axis. To this aim, we perform a second-order polynomial fitting of the two components of 
(a)

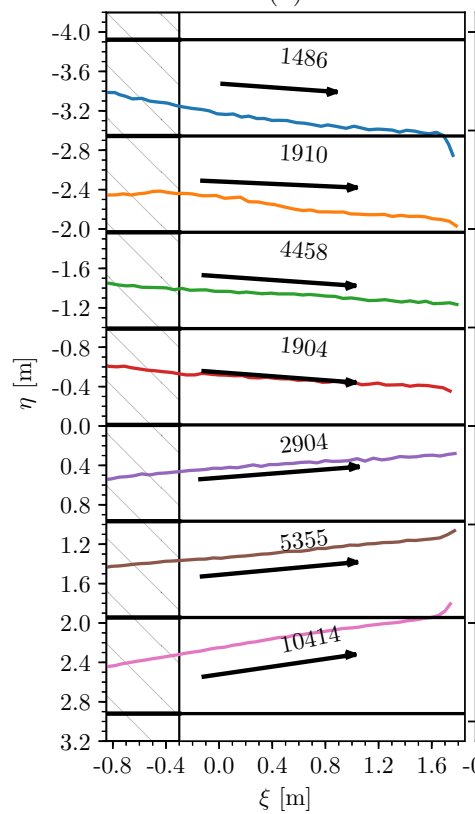

(b) - to City Center

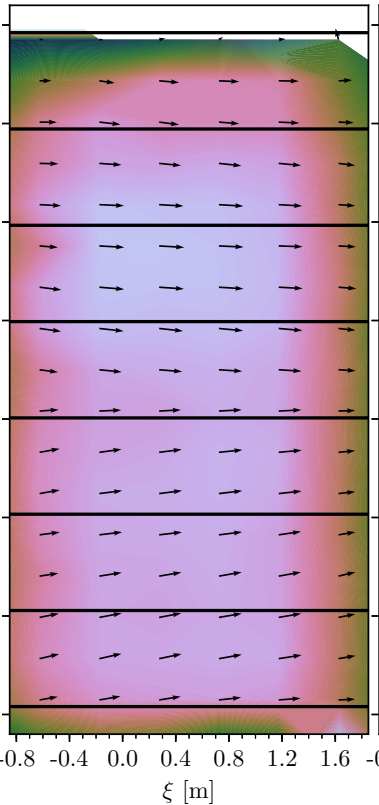

(c) - to Bus Terminal

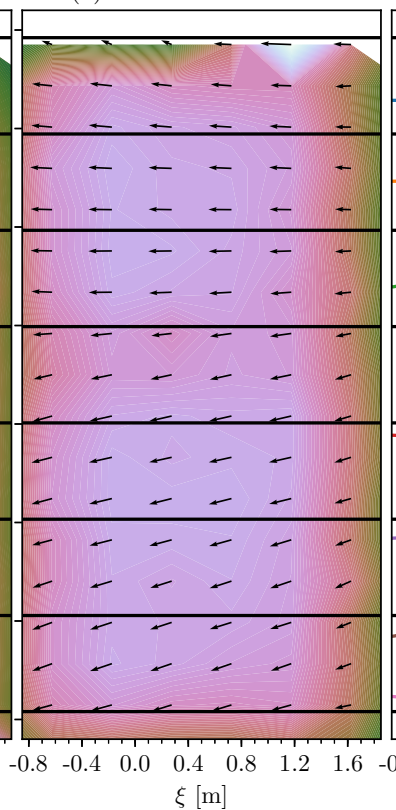

(d)

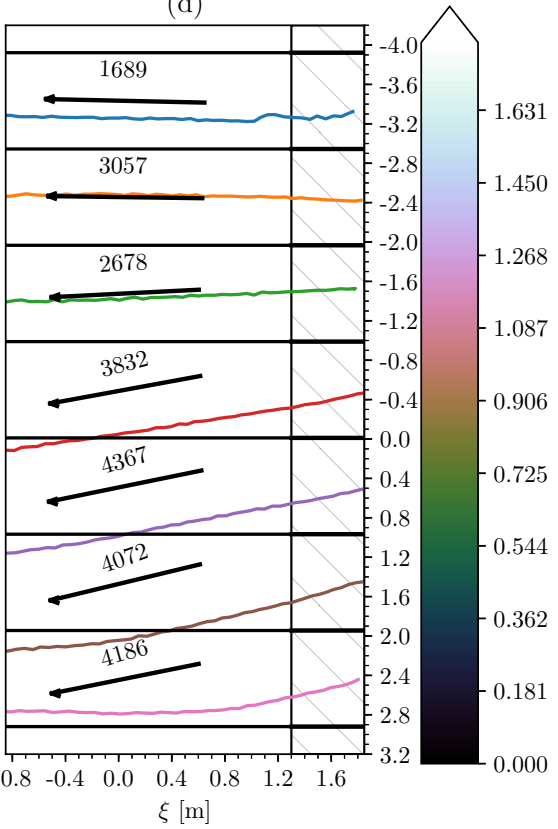

FIG. 14. Average (a) and (d) paths and (b) and (c) velocities of undisturbed pedestrians going to (a) and (b) the city center and (c) and (d) the bus terminal [cf. sketch in Fig. 2(a)]. Average paths (a) and (d) are computed by binning the trajectories by their initial points and then by averaging them in time (the number of trajectories per bin is reported). Average velocities (b) and (c) are computed by employing a grid of $10 \times 5$ tiles. The background colormap reports the average speed in $\mathrm{m} / \mathrm{s}$, according to the colorbar on the right.

each trajectory [in the horizontal-vertical reference of Fig. 14, say, $(\xi, \eta)]$ as a function of time. In formulas, we fit

$$
t \mapsto(\xi, \eta)
$$

via

$$
t \mapsto\left(a_{\xi} t^{2}+b_{\xi} t+c_{\xi}, a_{\eta} t^{2}+b_{\eta} t+c_{\eta}\right) .
$$

Hence, we rotate each trajectory to align the tangent line of the polynomial fit at $t=0$ to the $x$ axis, i.e.,

$$
(x, y)^{T}=\frac{1}{b_{\eta}^{2}+b_{\xi}^{2}}\left(\begin{array}{cc}
b_{\xi} & b_{\eta} \\
-b_{\eta} & b_{\xi}
\end{array}\right)(\xi, \eta)^{T} .
$$

Performing such a transformation, trajectories that are straight are simply aligned to the $x$ axis. In turn, $\mathrm{U}$-shaped trajectories, the most common example of inversion dynamics, get just their first (and possibly last) portion aligned with the $x$ axis.

\section{Quasirectilinear trajectories}

We use a similar fitting approach to distinguish trajectories that are quasirectilinear (straight but small fluctuations) from trajectories that exhibit large curvature or even U-turns. We split each trajectory in blocks of seven frames (i.e., about half a second, starting from the first frame) that we independently fit as in Eq. (B1) [see Fig. 15(a)]. In case the last block of a trajectory contains fewer than seven frames we neglect it. We estimate the angular slope of each block with reference to the horizontal direction as

$$
\phi=\left|\arctan \left(b_{\eta} / b_{\xi}\right)\right| .
$$

Rectilinear trajectories satisfy s.d. $(\phi) \approx 0^{+}$, where we evaluated the standard deviation (s.d.) on a block basis. In Fig. 15(b) we report the joint distribution of s.d. $(\phi)$ and the number of blocks per trajectory (i.e., a measure of the trajectory length). Neglecting the very short trajectories (the one-block case), we notice that most of our measurements lie in the low-s.d. $(\phi)$ region having between two and six blocks. These trajectories are mostly straight and encompass normal walking velocities. These feature Gaussian transversal fluctuations as discussed in Fig. 7(b).

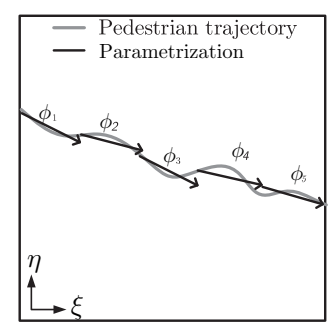

(a)

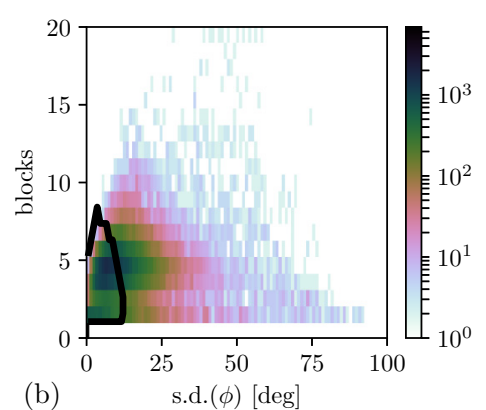

(b)
FIG. 15. (a) Analysis of the diluted motion assumes the trajectory to be a fluctuation around a straight intended path, which we call quasirectilinear. In order to isolate quasirectilinear trajectories, each track is divided into sets of seven contiguous frames (e.g., indexed by $i$ ). For each set the angle $\phi_{i}$ between the average velocity and the longitudinal direction in the corridor $(\xi)$ is evaluated. Quasirectilinear trajectories [as in (a)] feature low variance within the set $\left\{\phi_{i}\right\}$. Trajectories exhibiting instead significant drifts (high curvature, i.e., high $\left\{\phi_{i}\right\}$ variance) are neglected. (b) Joint distribution of s.d. $(\phi)[x$ axis; cf. Eq. (B2) $]$ and the number of blocks ( $y$ axis; one block gathers seven consecutive frames) for undisturbed pedestrians. The number of measurements per bin $\left(1^{\circ} \times 1\right.$ block $)$ follows the logarithmic colorbar. The region marked identifies the trajectories employed. 


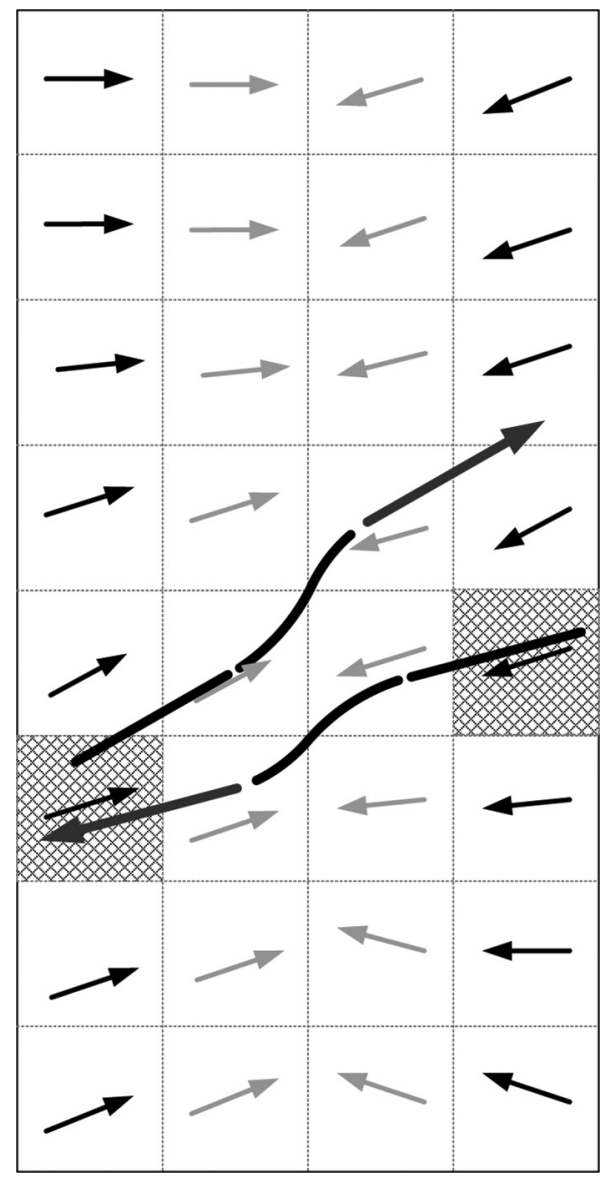

FIG. 16. Coordinate transformation for pedestrian pairs in avoidance for analysis convenience. Trajectories get rotated around the entrance point to compensate for intended paths not parallel to the corridor axis and then individually translated to conserve the minimum pedestrian distance. Compare the algorithm in Appendix C.

\section{APPENDIX C: COORDINATE TRANSFORMATION FOR PEDESTRIAN PAIRS IN AVOIDANCE}

In the analysis of pairwise avoidance interaction we performed a coordinate transformation, in similar spirit to Appendix $\mathrm{B}$, to bring the pairs of trajectories to a coordinate system convenient for the analysis and removed of average motions. The rationale for the transformation adheres to the following: (i) The minimum distance between pedestrians cannot be altered and (ii) the intended path of a pedestrian entering a given position is the same as an undisturbed pedestrian entering the same position. The deviations from such intended paths are what determines the $\Delta y$ variables. In algorithmic terms we proceeded as follows.
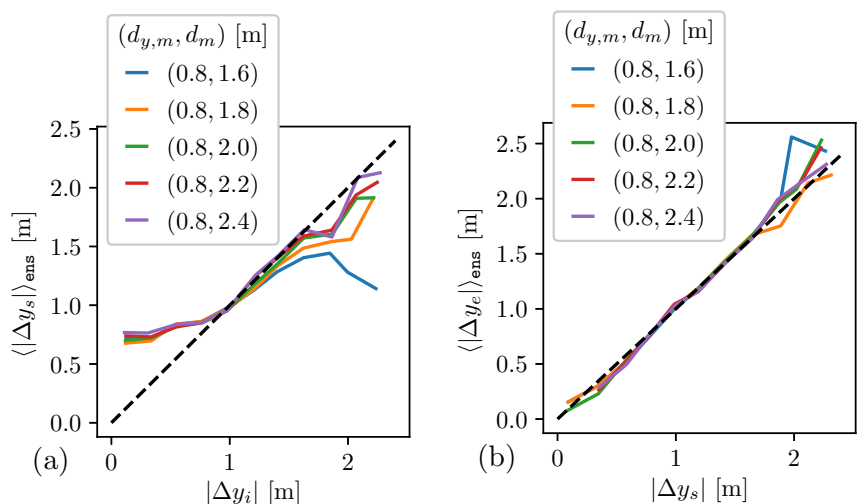

FIG. 17. Average transversal distance for cases (a) S1 and (b) S2 (cf. Sec. V) as a function of the parameter $d_{m}$ used to sparsify the graph $G$. (a) In case $\mathrm{S} 1$ a dependence on $d_{m}$ can be observed for $\left|\Delta y_{i}\right|>1 \mathrm{~m}$. Consistently with Sec. $\mathrm{V}$, as the region $\mathcal{I}_{p}$ grows in size, i.e., more and more pairs are included, the asymptotic behavior in Eq. (21) is recovered with increased accuracy. (b) In case S2 no particular dependence on the parameters is observed.

(1) Given a $(\xi, \eta)$ grid (coarse) as in Fig. 16, we calculated the average motion of undisturbed pedestrians directed both to the city center and to the bus terminal. We let $\theta^{1, C}(\xi, \eta)$ and $\theta^{1, T}(\xi, \eta)$ be the angles of the average velocity with the longitudinal axis of the corridor ( $\xi$ direction).

(2) Trajectories for pairs in interactions are rotated around their entering point $\left(\xi_{0}, \eta_{0}\right)$ of an angle $-\theta^{1, C}\left(\xi_{0}, \eta_{0}\right)$ or $-\theta^{1, T}\left(\xi_{0}, \eta_{0}\right)$ (dependent on the direction). This compensates for intended paths that are straight but not parallel to the $\xi$ axis to respect (ii).

(3) Trajectories are translated apart to respect (i).

The quantities $\Delta y_{i}, \Delta y_{s}$, and $\Delta y_{e}$ are finally calculated after these roto-translations.

\section{APPENDIX D: SENSITIVITY OF THE GRAPH-BASED SELECTION}

The parameters that we employed to sparsify the graph $G$ and select pairs of pedestrians avoiding each other have been chosen considering a typical size scale of the interaction. Nevertheless, it is reasonable to expect a sensitivity of the distributions in Figs. 10(a) and 11(a) with respect to these parameters. This sensitivity appears minimal, considering, for instance, average trends from Eq. (19). In Figs. 17(a) and 17(b), corresponding, respectively, to Figs. 10(a) and 11(a), we plot Eq. (19) dependent on the parameters $d_{m}$ and $d_{y, m}$. For computational reasons the data are restricted to only one day of measurements (27 November 2014).
[1] E. Cristiani, B. Piccoli, and A. Tosin, Multiscale Modeling of Pedestrian Dynamics (Springer International, Cham, 2014), Vol. 12.

[2] M. Moussaïd, D. Helbing, and G. Theraulaz, Proc. Natl. Acad. Sci. USA 108, 6884 (2011).

[3] M. Moussaïd, S. Garnier, G. Theraulaz, and D. Helbing, Top. Cogn. Sci. 1, 469 (2009).
[4] M. Moussaïd, D. Helbing, S. Garnier, A. Johansson, M. Combe, and G. Theraulaz, Proc. Biol. Sci. 276, 2755 (2009).

[5] P. Romanczuk, M. Bär, W. Ebeling, B. Lindner, and L. Schimansky-Geier, Eur. Phys. J. Spec. Top. 202, 1 (2012).

[6] N. Bellomo, B. Piccoli, and A. Tosin, Math. Models Methods Appl. Sci. 22, 1230004 (2012). 
[7] A. U. K. Wagoum, A. Seyfried, and S. Holl, Adv. Complex Syst. 15, 1250029 (2012).

[8] D. C. Duives, W. Daamen, and S. P. Hoogendoorn, Transport. Res. C: Emer. 37, 193 (2013).

[9] R. L. Hughes, Annu. Rev. Fluid Mech. 35, 169 (2003).

[10] D. Helbing and P. Molnár, Phys. Rev. E 51, 4282 (1995).

[11] M. C. Marchetti, J. F. Joanny, S. Ramaswamy, T. B. Liverpool, J. Prost, M. Rao, and R. A. Simha, Rev. Mod. Phys. 85, 1143 (2013).

[12] A. Corbetta, C.-m. Lee, R. Benzi, A. Muntean, and F. Toschi, Phys. Rev. E 95, 032316 (2017).

[13] M. Moussaïd, N. Perozo, S. Garnier, D. Helbing, and G. Theraulaz, PLoS ONE 5, e10047 (2010).

[14] A. Corbetta, J. Meeusen, C. Lee, and F. Toschi, Pedestrian and Evacuation Dynamics 2016 (University of Science and Technology of China Press, Hefei, 2016), pp. 18-24.

[15] T. Kretz, A. Grünebohm, M. Kaufman, F. Mazur, and M. Schreckenberg, J. Stat. Mech. (2006) P10001.

[16] J. Zhang and A. Seyfried, Proceedings of the 17th International IEEE Conference on Intelligent Transportation Systems (ITSC) (IEEE, Piscataway, 2014), pp. 542-547.

[17] F. Zanlungo, T. Ikeda, and T. Kanda, Phys. Rev. E 89, 012811 (2014).

[18] Y. Tamura, Y. Terada, A. Yamashita, and H. Asama, Int. J. Adv. Robot. Syst. 10, 310 (2013).

[19] M. Seitz, S. Seer, S. Klettner, O. Handel, and G. Köster, in Traffic and Granular Flows '15, edited by W. Daamen and V. Knoop (Springer, Berlin, 2016).

[20] S. Kosiantis, I. Zaharakis, and P. Pintelas, in Emerging Artificial Intelligence Applications in Computer Engineering, edited by I. Maglogiannis, K. Karpouzis, and B. A. Wallace (IOS, Amsterdam, 2007), Vol. 160, pp. 3-24.

[21] W. T. Coffey, Y. P. Kalmykov, and J. T. Waldron, The Langevin Equation: With Applications to Stochastic Problems in Physics, Chemistry and Electrical Engineering (World Scientific, Singapore, 2004).
[22] Kinect for Xbox 360 (Microsoft Corp., Redmond, 2012).

[23] A. Corbetta, L. Bruno, A. Muntean, and F. Toschi, Transp. Res. Proc. 2, 96 (2014).

[24] A. Corbetta, C. Lee, A. Muntean, and F. Toschi, in Traffic and Granular Flows '15 (Ref. [19]), Chap. 7.

[25] S. Seer, N. Brändle, and C. Ratti, Transport. Res. C-Emer. 48, 212 (2014).

[26] D. Brščić, T. Kanda, T. Ikeda, and T. Miyashita, IEEE Trans. Human-Mach. Syst. 43, 522 (2013).

[27] A. Corbetta, C.-m. Lee, A. Muntean, and F. Toschi, Collect. Dyn. 1, 1 (2017).

[28] D. Helbing and A. Johansson, in Encyclopedia of Complexity and Systems Science, edited by R. A. Meyers (Springer, New York, 2009), Vol. 16, pp. 6476-6495.

[29] M. Ballerini, N. Cabibbo, R. Candelier, A. Cavagna, E. Cisbani, I. Giardina, V. Lecomte, A. Orlandi, G. Parisi, A. Procaccini, M. Viale, and V. Zdravkovic, Proc. Natl. Acad. Sci. USA 105, 1232 (2008).

[30] G. Arechavaleta, J. Laumond, H. Hicheur, and A. Berthoz, IEEE Trans. Robot. 24, 5 (2008).

[31] D. R. Parisi, M. Gilman, and H. Moldovan, Physica A 388, 3600 (2009).

[32] A. Corbetta, Ph.D. thesis, Technische Universiteit Eindhoven, 2016.

[33] A. Corbetta, A. Muntean, and K. Vafayi, Math. Biosci. Eng. 12, 337 (2015).

[34] J. Willneff, Ph.D. thesis, Institut für Geodäsie und Photogrammetrie an der Eidgenössichen Technischen Hochschule, 2003.

[35] The OpenPTV Consortium, OpenPTV: Open source particle tracking velocimetry, available at http://www.openptv.net/ (2012).

[36] A. Savitzky and M. J. Golay, Anal. Chem. 36, 1627 (1964).

[37] U. Gülan, B. Lüthi, M. Holzner, A. Liberzon, A. Tsinober, and W. Kinzelbach, Exp. Fluids 53, 1469 (2012). 